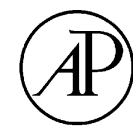

ACADEMIC

PRESS

\title{
Algebraic solution of the supersymmetric hydrogen atom in $d$ dimensions
}

\author{
A. Kirchberg, ${ }^{a}$ J.D. Länge, ${ }^{a, *}$ P.A.G. Pisani, ${ }^{b}$ and A. Wipf ${ }^{a}$ \\ ${ }^{a}$ Theoretisch-Physikalisches Institut, Friedrich Schiller Universität Jena, Fröbelstieg 1, \\ 07743 Jena, Germany \\ ${ }^{\mathrm{b}}$ IFLP, Departamento de Física de Ciencias Exactas, UNLP C.C. 67, 1900 La Plata, Argentina
}

Received 19 September 2002

\begin{abstract}
In this paper the $\mathscr{N}=2$ supersymmetric extension of the Schrödinger Hamiltonian with $1 / r$-potential in arbitrary space-dimensions is constructed. The supersymmetric hydrogen atom admits a conserved Laplace-Runge-Lenz vector which extends the rotational symmetry $S O(d)$ to a hidden $S O(d+1)$ symmetry. This symmetry of the system is used to determine the discrete eigenvalues with their degeneracies and the corresponding bound state wave functions.

(c) 2003 Elsevier Science (USA). All rights reserved.
\end{abstract}

Keywords: Laplace-Runge-Lenz vector; Supersymmetric quantum mechanics; Dynamical symmetry; Hydrogen atom

\section{Introduction}

For a closed system of two non-relativistic point masses interacting via a central force the angular momentum $\mathbf{L}$ of the relative motion is conserved and the motion is always in the plane perpendicular to $\mathbf{L}$. If the force is derived from the Newton or Coulomb potential, there is an additional conserved quantity: the Laplace-RungeLenz ${ }^{1}$ vector [1]. For the hydrogen atom this vector has the form

\footnotetext{
* Corresponding author.

E-mail addresses: a.kirchberg@tpi.uni-jena.de (A. Kirchberg), J.D.Laenge@tpi.uni-jena.de (J.D. Länge), Pisani@obelix.fisica.unlp.edu.ar (P.A.G. Pisani), A.Wipf@tpi.uni-jena.de (A. Wipf).

${ }^{1}$ A more suitable name for this constant of motion would be Hermann-Bernoulli-Laplace vector, see [2]. 


$$
\mathbf{C}=\frac{1}{m} \mathbf{p} \times \mathbf{L}-\frac{e^{2}}{r} \mathbf{r}, \quad \mathbf{L}=\mathbf{r} \times \mathbf{p},
$$

where $m$ denotes the reduced mass of the proton-electron system. The LaplaceRunge-Lenz vector is perpendicular to $\mathbf{L}$ and hence is a vector in the plane of the orbit. It points in the direction of the semi-major axis.

Quantum mechanically, one defines the hermitian Laplace-Runge-Lenz vector

$$
\mathbf{C}=\frac{1}{2 m}(\mathbf{p} \times \mathbf{L}-\mathbf{L} \times \mathbf{p})-\frac{e^{2}}{r} \mathbf{r} .
$$

By exploiting the existence of this conserved vector operator, Pauli calculated the spectrum of the hydrogen atom by purely algebraic means $[3,4]$. He noticed that the angular momentum $\mathbf{L}$ together with the vector

$$
\mathbf{K}=\sqrt{\frac{-m}{2 H} \mathbf{C}},
$$

which is well defined and hermitian on bound states with negative energies, generate a hidden $S O(4)$ symmetry algebra

$$
\left[L_{a}, L_{b}\right]=\mathrm{i} \hbar \epsilon_{a b c} L_{\mathrm{c}}, \quad\left[L_{a}, K_{b}\right]=\mathrm{i} \hbar \epsilon_{a b c} K_{\mathrm{c}}, \quad\left[K_{a}, K_{b}\right]=\mathrm{i} \hbar \epsilon_{a b c} L_{\mathrm{c}},
$$

and that the Hamiltonian can be expressed in terms of $\mathbf{K}^{2}+\mathbf{L}^{2}$, one of the two second-order Casimir operators of this algebra, as follows:

$$
H=-\frac{m e^{4}}{2} \frac{1}{\mathbf{K}^{2}+\mathbf{L}^{2}+\hbar^{2}} .
$$

One further observes that the other Casimir operator $\mathbf{K} \cdot \mathbf{L}$ vanishes and arrives at the bound state energies by purely group theoretical methods. The existence of the conserved vector $\mathbf{K}$ also explains the accidental degeneracy of the hydrogen spectrum. Only much later the scattering amplitude of the hydrogen atom has been calculated with the help of the Laplace-Runge-Lenz vector [5].

In this paper we shall generalize these results in two directions: first to the hydrogen atom in arbitrary dimensions ${ }^{2}[21]$ and second to the corresponding supersymmetric extensions. In Section 2 we prove that the Schrödinger Hamiltonian in $d$ dimensions with $1 / r$-potential admits a generalization of the Laplace-Runge-Lenz vector. Together with the generators of the rotation group $S O(d)$ this vector generates the dynamical symmetry group $S O(d+1)$. This hidden symmetry allows then for a purely algebraic solution of the hydrogen atom in arbitrary dimensions, very much as in three dimensions.

In Section 3 we summarize the extensions of $d$-dimensional Schrödinger Hamiltonians to models with $\mathscr{N}=2$ supersymmetry. The corresponding Hamiltonians may be written as $2^{d} \times 2^{d}$-dimensional matrix Schrödinger operators. For a particular choice of the superpotential we obtain the supersymmetric extension of the hydrogen atom. For instance in $d=3$ (and in a suitable basis) we find the Hamiltonian

\footnotetext{
${ }^{2}$ When speaking of the $d$-dimensional hydrogen atom, we always mean the $1 / r$-potential, although this potential permits the application of Gauss' law in three dimensions only.
} 


$$
H=\left(p^{2}+\lambda^{2}\right) 1_{8}-\frac{2 \lambda}{r}\left(\begin{array}{llll}
1 & & & \\
& M_{1} & & \\
& & M_{2} & \\
& & & -1
\end{array}\right),
$$

with $3 \times 3$ matrices

$$
M_{1 a b}=\hat{x}_{a} \hat{x}_{b}, \quad M_{2 a b}=\delta_{a b}-(-)^{a+b} \hat{x}_{a} \hat{x}_{b} .
$$

In Section 4 we construct the supersymmetric extensions of the angular momentum and the Laplace-Runge-Lenz vector. Similarly as for the purely bosonic system, together they form a dynamical $S O(d+1)$ symmetry algebra. This symmetry is exploited in Section 2 to obtain the discrete eigenvalues and their degeneracies. In Section 6 we characterize all bound state wave functions. In Section 7 we illustrate our general results by analyzing in detail the supersymmetric hydrogen atoms in two, three, and four dimensions.

The appendices contain the necessary group theoretical tools needed in the main body of the paper.

\section{The coulomb problem and its symmetries in $d$ dimensions}

We generalize the Coulomb problem to arbitrary dimensions by keeping the $1 / r$ potential, although this potential solves the Poisson equation in three dimensions only. With this assumption the hydrogen atom in $d$ dimensions is governed by the Schrödinger equation

$$
H \psi=\left(-\frac{\hbar^{2}}{2 m} \Delta-\frac{e^{2}}{r}\right) \psi=E \psi .
$$

It is convenient to measure distances in units of the Compton wavelength $\chi_{\mathrm{c}}=\hbar / \mathrm{mc}$. With respect to these dimensionless coordinates Eq. (3) takes the simpler form

$$
H \psi=E \psi, \quad H=p^{2}-\frac{\eta}{r}, \quad p_{a}=\frac{1}{\mathrm{i}} \partial_{a}, \quad a=1, \ldots, d,
$$

where $\eta$ is twice the fine structure constant $\alpha$ and the dimensionless energy $E$ is measured in units of $m c^{2} / 2$. The central force is attractive for positive $\eta$.

The hermitian generators $L_{a b}=x_{a} p_{b}-x_{b} p_{a}$ of the rotation group in $d$ dimensions satisfy the familiar $s o(d)$ commutation relations

$$
\left[L_{a b}, L_{c d}\right]=\mathrm{i}\left(\delta_{a c} L_{b d}+\delta_{b d} L_{a c}-\delta_{a d} L_{b c}-\delta_{b c} L_{a d}\right),
$$

where indices run from 1 to $d$. It is not very difficult to guess the generalization of the Laplace-Runge-Lenz vector (1) in $d$ dimensions [21]

$$
C_{a}=L_{a b} p_{b}+p_{b} L_{a b}-\frac{\eta x_{a}}{r} .
$$

Indeed, these operators commute with the Hamiltonian (4). They form a $S O(d)$ vector, 


$$
\left[L_{a b}, C_{\mathrm{c}}\right]=\mathrm{i}\left(\delta_{a c} C_{b}-\delta_{b c} C_{a}\right),
$$

and the commutator of $C_{a}$ and $C_{b}$ is proportional to the angular momentum

$$
\left[C_{a}, C_{b}\right]=-4 \mathrm{i} L_{a b} H .
$$

Now we may proceed as we did in three dimensions and define on the negative energy $(E<0)$ subspace of the Hilbert space $\mathscr{H}=L_{2}\left(\mathbb{R}^{d}\right)$ the hermitian operators

$$
K_{a}=\frac{1}{\sqrt{-4 H}} C_{a} \quad \text { with } \quad\left[K_{a}, K_{b}\right]=\mathrm{i} L_{a b} .
$$

The operators $\left\{L_{a b}, K_{a}\right\}$ form a closed symmetry algebra of dimension $(d+1) d / 2$. More explicitly, they can be combined to form generators $L_{A B}$ of the orthogonal $\operatorname{group}^{3} S O(d+1)$

$$
L_{A B}=\left(\begin{array}{c|c}
L_{a b} & K_{a} \\
\hline-K_{b} & 0
\end{array}\right),
$$

which implies that the $L_{A B}$ obey the commutation relations (5) with indices running from 1 to $d+1$. We can verify a relation similar to (2) by solving

$$
C_{a} C_{a}=-4 K_{a} K_{a} H=\eta^{2}+\left(2 L_{a b} L_{a b}+(d-1)^{2}\right) H
$$

for $H$. We obtain the Hamiltonian

$$
H=p^{2}-\frac{\eta}{r}=-\frac{\eta^{2}}{(d-1)^{2}+4 \mathscr{C}_{(2)}}
$$

in terms of the second-order Casimir operator of the dynamical symmetry group $S O(d+1)$,

$$
\mathscr{C}_{(2)}=\frac{1}{2} L_{A B} L_{A B}=\frac{1}{2} L_{a b} L_{a b}+K_{a} K_{a} .
$$

This is the generalization of (2) we have been looking for. It remains to characterize those irreducible representations of $S O(d+1)$ which are realized in the Hilbert space $L_{2}\left(\mathbb{R}^{d}\right)$. In three dimensions the allowed representations are fixed by the condition $\mathbf{K} \cdot \mathbf{L}=0$ on the second Casimir operator of $S O(4)$. We expect $n-1$ conditions on the $n$ Casimir operators of the dynamical symmetry group $S O(2 n+1)$ in $d=2 n$ dimensions and $n$ conditions on the $n+1$ Casimir operators of $S O(2 n+2)$ in $d=2 n+1$ dimensions. In the following we treat the even- and odd-dimensional cases separately.

Even-dimensional spaces: An irreducible representation is uniquely characterized by its highest weight state. By definition, this state is annihilated by all raising operators belonging to the simple roots. To characterize these states one conveniently chooses complex coordinates $z_{1}, \ldots, z_{n}$ in $\mathbb{R}^{2 n}$ such that the raising operators of the

\footnotetext{
${ }^{3}$ For scattering states $(E>0)$ a similar redefinition leads to generators of the Lorentz group $S O(1, d)$. Here we are interested in bound states and will not further discuss this possibility.
} 
dynamical symmetry group with generators $J_{A B}$ in (9) have the simple form (cf. Appendix A)

$$
\begin{aligned}
& E_{i}=\frac{1}{\mathrm{i}}\left(z_{i} \partial_{i+1}-\bar{z}_{i+1} \bar{\partial}_{i}\right), \quad i=1, \ldots, n-1, \\
& E_{n}=\frac{1}{\sqrt{2}}\left(C_{d-1}+\mathrm{i} C_{d}\right)=-2 z_{n} \Delta+\left(2 r \partial_{r}+d-1\right) \bar{\partial}_{n}-\frac{\eta z_{n}}{r} .
\end{aligned}
$$

In the formula for $E_{n}$ we actually should have used the operators $K_{d-1}$ and $K_{d}$ in (8), since they appear as components of $J_{A B}$. But since we are only interested in highest weight states which are annihilated by $E_{n}$ we may take the operators $C_{d-1}$ and $C_{d}$ instead. Also note that all simple roots of $S O(d)$ are positive roots of $S O(d+1)$ so that all highest weight states of $S O(d+1)$ are automatically highest weight states of $S O(d) \subset S O(d+1)$. Now it is not difficult to see that a regular wave function which is annihilated by all simple raising operators of $S O(d)$, that is by the $n-1$ operators in (12) and by the operator $z_{n-1} \bar{\partial}_{n}-z_{n} \bar{\partial}_{n-1}$ (see Appendix A.1), must have the form (cf. (A.10))

$$
\Psi=f(r) z_{1}^{\ell} .
$$

It is a highest weight state of $S O(d+1)$, if in addition it is annihilated by $E_{n}$ in (13)

$$
E_{n} \Psi=-\left\{(d-1+2 \ell) \frac{\mathrm{d}}{\mathrm{d} r} \log f+\eta\right\} \frac{z_{n}}{r} \Psi=0 .
$$

Hence the highest weight state reads

$$
\Psi=\mathrm{e}^{-\gamma_{\ell} r} z_{1}^{\ell}, \quad \gamma_{\ell}=\frac{\eta}{d-1+2 \ell} .
$$

The constant $\eta$ must be positive for bound states to exist. $\Psi$ is of course an eigenfunction of all $n$ Cartan generators $H_{i}=z_{i} \partial_{i}-\bar{z}_{i} \bar{\partial}_{i}$ of $S O(2 n+1)$ with eigenvalues $(\ell, 0, \ldots, 0)$. That shows that only the symmetric multiplets of the dynamical symmetry group appear. ${ }^{4}$ From (A.9) we take the values of the second-order Casimir operator for symmetric multiplets

$$
\mathscr{C}_{(2)}=\ell(\ell+d-1), \quad \ell=0,1,2, \ldots,
$$

and their dimensions

$$
\operatorname{dim} V_{\ell}=\left(\begin{array}{c}
\ell+d \\
\ell
\end{array}\right)-\left(\begin{array}{c}
\ell+d-2 \\
\ell-2
\end{array}\right) .
$$

The $\operatorname{dim} V_{\ell}$ states of the symmetric representation are obtained by acting repeatedly with the lowering operators

$$
E_{i<n}^{\dagger}=\frac{1}{\mathrm{i}}\left(z_{i+1} \partial_{i}-\bar{z}_{i} \bar{\partial}_{i+1}\right) \quad \text { and } \quad E_{n}^{\dagger}=-2 \bar{z}_{n} \Delta+\partial_{n}\left(2 r \partial_{r}-1\right)-\frac{\eta \bar{z}_{n}}{r}
$$

on the state (15). This way one obtains all bound $H$-eigenstates with the same energy.

\footnotetext{
${ }^{4}$ This corresponds to the extension of Fock's method to $d$ dimensions, cf. $[4,6,7]$.
} 
Odd-dimensional spaces: For $d=2 n+1$ the rank of the dynamical symmetry group $S O(d+1)$ exceeds the rank of the rotation group $S O(d)$ by 1 . We combine the first $2 n$ coordinates to $n$ complex ones and take coordinates $z_{1}, \ldots, z_{n}, x_{d}$ in $\mathbb{R}^{d}$, see Appendix A.2.

As Cartan generators we choose

$$
H_{i}=z_{i} \partial_{i}-\bar{z}_{i} \bar{\partial}_{i}, \quad i=1, \ldots, n \text { and } H_{n+1}=C_{d} .
$$

The raising operators are the $n+1$ operators $E_{i}$ in (12) supplemented by

$$
E_{n}=\frac{1}{\mathrm{i}}\left(z_{n} \partial_{x_{d}}-x_{d} \bar{\partial}_{z_{n}}\right) \text { and } \quad E_{n+1}=\frac{1}{\sqrt{2}}\left(C_{d-2}+\mathrm{i} C_{d-1}\right) .
$$

The last raising operator $E_{n+1}$ coincides with $E_{n}$ in (13). A regular wave function is annihilated by the first $n$ raising operators only if it has the form $\Psi=f(r) z_{1}^{\ell}$. The requirement that it is annihilated by the last raising operator $E_{n+1}$ again leads to Eq. (14) and hence to the solution $\Psi$ in (15). To determine the multiplets with this highest weight state we need to calculate the highest weight vector, that is the value of the Cartan generators on $\Psi$. Clearly,

$$
H_{1} \Psi=\ell \Psi \quad \text { and } \quad H_{i} \Psi=0 \text { for } i=2, \ldots, n .
$$

The last Cartan generator in (18) has the explicit form

$$
H_{n+1}=-2 x_{d} \Delta+\left(2 r \partial_{r}+d-1\right) \partial_{x_{d}}-\frac{\eta x_{d}}{r}
$$

and we find

$$
H_{n+1} \Psi=-\left\{(d-1+2 \ell) \frac{\mathrm{d}}{\mathrm{d} r} \log f+\eta\right\} \frac{x_{d}}{r} \Psi=0,
$$

after using $f=\mathrm{e}^{-\gamma_{\ell} r}$. Hence, on any highest weight state the operators $H_{2}, \ldots, H_{n+1}$ vanish and again we find the totally symmetric representations of the dynamical symmetry group $S O(d+1)$. The eigenvalues of the second-order Casimir operator and the dimension of the representations are given by the same formulae (16), (17) as for the hydrogen atom in even dimensions.

Since $\eta$ is twice the fine structure constant $\alpha=e^{2} / \hbar c$ and $E$ is measured in units of $m c^{2} / 2$ formula (11) yields the well-known [8] bound state energies in three dimensions

$$
E_{\ell}=-\frac{\alpha^{2}}{1+\ell(\ell+2)} \frac{m c^{2}}{2}=-\frac{m e^{4}}{2 \hbar^{2}} \frac{1}{n^{2}} \equiv E_{n}, \quad n=1+\ell=1,2, \ldots
$$

The degeneracy of $E_{n}$ is the dimension $n^{2}$ of the corresponding symmetric representation of $S O(4)$. All $n^{2}$ states with the same energy $E_{n}$ are gotten by acting with the two lowering operators on the highest weight state

$$
\Psi(x)=\mathrm{e}^{-\gamma_{n} r}\left(x_{1}+\mathrm{i} x_{2}\right)^{\ell}, \quad \gamma_{n}=\frac{\alpha}{n} .
$$


In $d$ dimensions the corresponding formulae read

$$
E_{\ell}=-\frac{m e^{4}}{2 \hbar^{2}}\left(\ell+\frac{d-1}{2}\right)^{-2}, \quad \Psi(x)=\mathrm{e}^{-\gamma_{\ell} r}\left(x_{1}+\mathrm{i} x_{2}\right)^{\ell}, \quad \gamma_{\ell}=\frac{\alpha}{\ell+(d-1) / 2},
$$

and $E_{\ell}$ has degeneracy $\operatorname{dim} V_{\ell}$ in (17).

The appearance of the accidental degeneracy - phrased in the language of representation theory - corresponds to the following branching rule: the totally symmetric representations of the dynamical symmetry group $S O(d+1)$ branches into those completely symmetric representations of the rotation group $S O(d)$ with equal or shorter Young diagrams

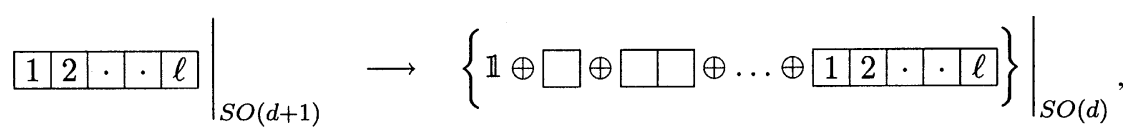

all of them possessing the same energy. The energy, its degeneracy and the bound state wave functions are uniquely fixed by the representation of the dynamical symmetry group. Every totally symmetric representation of $S O(d+1)$ appears once and only once and corresponds to the multiplet with energy $E_{\ell}$. The angular momentum content of this multiplet is determined by the branching rule (19). In three dimensions this expresses just the well-known fact that for each value of $E_{n=\ell+1}$ the orbital angular momentum can vary from 0 to $n-1=\ell$.

\section{3. $\mathscr{N}=2$ supersymmetric quantum mechanics}

We wish to further generalize our results to the supersymmetric hydrogen atom in $d$ dimensions. For that purpose we need a supersymmetric extension of $d$-dimensional Schrödinger operators and in particular of the operator in (4). Such supersymmetric Hamiltonians can be written as

$$
H=\left\{Q, Q^{\dagger}\right\}=H^{\dagger} \quad \text { with } Q^{2}=Q^{\dagger 2}=0,
$$

where the supercharge $Q$ and its adjoint $Q^{\dagger}$ anticommute with a self-adjoint idempotent operator $\Gamma$. The subspace on which $\Gamma=1$ is called the bosonic sector and its complement the fermionic sector. Hence, $Q$ transforms bosons into fermions and vice versa. From (20) one sees at once that the supercharge commutes with the supersymmetric Hamiltonian,

$$
[Q, H]=0,
$$

i.e., generates a supersymmetry of the system. The simplest models exhibiting the structure (20) are $2 \times 2$-dimensional matrix Schrödinger operators in one dimension. Such models were first studied by Nicolai and Witten [9-11].

Supersymmetric Hamiltonians in higher dimensions have been introduced previously by several authors $[12,13]$. Here we briefly present the construction used in this paper. We introduce a set of fermionic creation and annihilation operators, 


$$
\left\{\psi_{a}, \psi_{b}^{\dagger}\right\}=\delta_{a b}, \quad\left\{\psi_{a}, \psi_{b}\right\}=\left\{\psi_{a}^{\dagger}, \psi_{b}^{\dagger}\right\}=0, a, b=1, \ldots, d,
$$

and the Fock space with vacuum $|0\rangle$ which is annihilated by all operators $\psi_{a}$. This space splits into sub-spaces,

$$
\mathscr{C}=\mathscr{C}_{0} \oplus \mathscr{C}_{1} \oplus \cdots \oplus \mathscr{C}_{d}, \quad \operatorname{dim} \mathscr{C}_{p}=\left(\begin{array}{l}
d \\
p
\end{array}\right), \quad \operatorname{dim} \mathscr{C}=2^{d},
$$

labeled by their 'fermion number'

$$
\left.N\right|_{\mathscr{C}_{p}}=p 1, \quad \text { where } N=\sum_{a=1}^{d} \psi_{a}^{\dagger} \psi_{a} .
$$

As basis in $\mathscr{C}_{p}$ we may choose

$$
\left|a_{1} \ldots a_{p}\right\rangle=\psi_{a_{1}}^{\dagger} \ldots \psi_{a_{p}}^{\dagger}|0\rangle, \quad a_{1}<a_{2}<\cdots<a_{p} .
$$

Along with $\mathscr{C}$ the Hilbert space of all square integrable wave functions decomposes as

$$
\mathscr{H}=\mathscr{H}_{0} \oplus \mathscr{H}_{1} \oplus \cdots \oplus \mathscr{H}_{d}, \quad \text { where }\left.N\right|_{\mathscr{H}_{p}}=p \mathbb{1} .
$$

An arbitrary wave function in $\mathscr{H}_{p}$ has the expansion

$$
\Psi=f_{a_{1} \ldots a_{p}}(x)\left|a_{1} \ldots a_{p}\right\rangle, \quad f_{a_{1} \ldots a_{p}} \text { totally antisymmetric. }
$$

An explicit realization of the creation and annihilation operators can be given in terms of the hermitian $\gamma$-matrices in $2 d$ Euclidean dimensions: $\psi_{a}=1 / 2\left(\gamma_{a}-\mathrm{i} \gamma_{d+a}\right)$.

The supercharge and its adjoint ${ }^{5}$ are defined via

$$
\begin{aligned}
& Q=\mathrm{e}^{-\chi} Q_{0} \mathrm{e}^{\chi}=\mathrm{i} \sum_{a} \psi_{a}\left(\partial_{a}+\partial_{a} \chi\right) \text { with } Q_{0}=\mathrm{i} \psi_{a} \partial_{a}, \\
& Q^{\dagger}=\mathrm{e}^{\chi} Q_{0}^{\dagger} \mathrm{e}^{-\chi}=\mathrm{i} \sum_{a} \psi_{a}^{\dagger}\left(\partial_{a}-\partial_{a} \chi\right) \quad \text { with } Q_{0}^{\dagger}=\mathrm{i} \psi \psi_{a}^{\dagger} \partial_{a} .
\end{aligned}
$$

At this point the real superpotential $\chi\left(x_{1}, \ldots, x_{d}\right)$ remains unspecified. From (21) it follows at once that the free supercharge $Q_{0}$ is nilpotent and since $Q$ and $Q_{0}$ are related by a similarity transformation the same holds true for $Q$. The supercharge $Q$ only contains annihilation operators and hence decreases the fermion number by 1 . Its adjoint $Q^{\dagger}$ increases it by 1 ,

$$
[N, Q]=-Q \quad \text { and } \quad\left[N, Q^{\dagger}\right]=Q^{\dagger} .
$$

The supersymmetric Hamiltonian defined in (20) is a $2^{d} \times 2^{d}$-dimensional matrix Schrödinger operator and takes the following form (cf. also [12,13]):

\footnotetext{
${ }^{5}$ The hermitian linear combinations $Q_{1}=Q+Q^{\dagger}$ and $Q_{2}=\mathrm{i}\left(Q-Q^{\dagger}\right)$ satisfy the standard $\mathscr{N}=2$ supersymmetry algebra $\left\{Q_{i}, Q_{j}\right\}=2 H \delta_{i j}$.
} 


$$
H=\left\{-\Delta+(\nabla \chi, \nabla \chi)+\Delta_{\chi}\right\} \mathbb{1}_{2^{d}}-2 \sum_{a, b=1}^{d} \psi_{a}^{\dagger} \chi_{a b} \psi_{b}, \quad \chi_{a b}=\frac{\partial^{2} \chi}{\partial x_{a} \partial x_{b}}
$$

We use brackets to indicate contraction of indices as $(A, B)=\sum_{a} A^{a} B^{a}$. Contrary to the supercharge and its adjoint the supersymmetric Hamiltonian $H$ commutes with the number operator $N$ and hence leaves each subspace $\mathscr{H}_{p}$ in the decomposition (23) invariant

$$
H: \mathscr{H}_{p} \rightarrow \mathscr{H}_{p} .
$$

On the subspace $\mathscr{H}_{p}$ the supersymmetric Hamiltonian is still a matrix Schrödinger operator

$$
\left.H\right|_{\mathscr{H}_{p}}=-\Delta 1+V^{(p)}, \quad \operatorname{tr} \mathbb{1}=\left(\begin{array}{l}
d \\
p
\end{array}\right) .
$$

Only in the extreme sectors $\mathscr{H}_{0}$ and $\mathscr{H}_{p}$ do we get ordinary Schrödinger operators acting on one-component wave functions. With

$$
\psi_{a}^{\dagger} \psi_{b}|0\rangle=0 \quad \text { and } \quad \psi_{a}^{\dagger} \psi_{b}|12 \ldots d\rangle=\delta_{a b}|12 \ldots d\rangle
$$

the corresponding potentials take the form

$$
V^{(0)}=(\nabla \chi, \nabla \chi)+\Delta \chi \quad \text { and } \quad V^{(d)}=(\nabla \chi, \nabla \chi)-\Delta \chi
$$

More generally, for an arbitrary state $\Psi=f_{a_{1} \ldots a_{p}}\left|a_{1} \ldots a_{p}\right\rangle \in \mathscr{H}_{p}$ the Hamiltonian acts as follows:

$$
\left\langle a_{1} \ldots a_{p} \mid H \Psi\right\rangle=\left(-\Delta+V^{(0)}\right) f_{a_{1} \ldots a_{p}}+2 \sum_{b, i=1}^{p}(-)^{i} \chi_{a_{i} b} f_{b a_{1} \ldots \check{a}_{i} \ldots a_{p}},
$$

where $\check{a}_{i}$ indicates that $a_{i}$ us omitted. The nilpotent supercharges give rise to the following Hodge-type decomposition of the Hilbert space:

$$
\mathscr{H}=Q \mathscr{H} \oplus Q^{\dagger} \mathscr{H} \oplus \operatorname{Ker} H,
$$

where the finite-dimensional subspace $\operatorname{Ker} H$ is spanned by the zero modes of $H$. Indeed, on the orthogonal complement of $\operatorname{Ker} H$ we may invert $H$ and write

$$
\mathscr{H}_{0}^{\perp}=\left(Q Q^{\dagger}+Q^{\dagger} Q\right) H^{-1} \mathscr{H}_{0}^{\perp}=Q\left(\frac{Q^{\dagger}}{H} \mathscr{H}_{0}^{\perp}\right)+Q^{\dagger}\left(\frac{Q}{H} \mathscr{H}_{0}^{\perp}\right),
$$

which proves (28). The supercharge $Q$ maps every energy-eigenstate in $Q^{\dagger} \mathscr{H} \cap \mathscr{H}_{p}$ with positive energy into an eigenstate in $Q \mathscr{H} \cap \mathscr{H}_{p-1}$ with the same energy. Its adjoint maps eigenstates in $Q \mathscr{H} \cap \mathscr{H}_{p}$ into those in $Q^{\dagger} \mathscr{H} \cap \mathscr{H}_{p+1}$ with the same energy. With the exception of the zero energy states there is an exact pairing between the eigenstates and energies in the bosonic and in the fermionic sector as depicted below: 


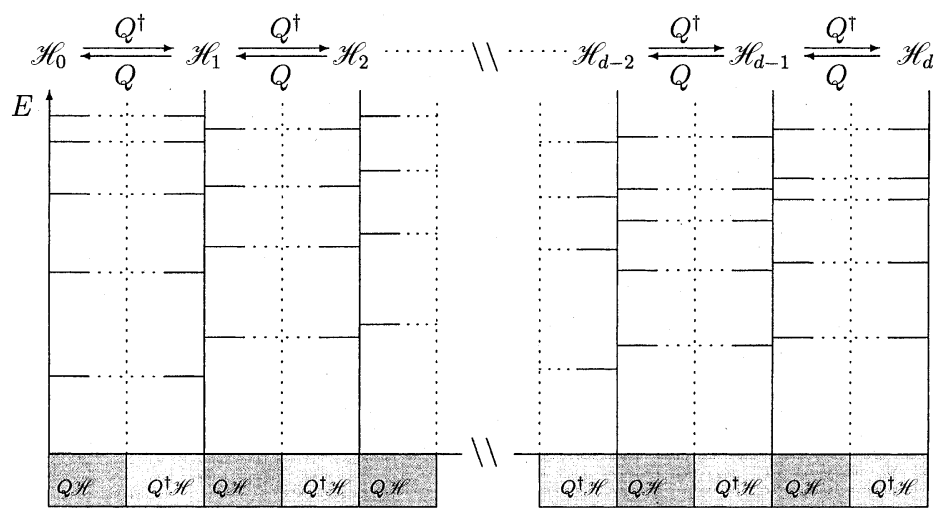

The supersymmetric systems with supercharges (25) admit a generalized Poincaré duality relating $\mathscr{H}_{d-p}$ with $\mathscr{H}_{p}$. This is seen as follows: instead of the vacuum $|0\rangle \in \mathscr{H}_{0}$, which is annihilated by all $\psi_{a}$, we take || 0$\rangle=\psi_{1}^{\dagger} \ldots \psi_{d}^{\dagger}|0\rangle \in \mathscr{H}_{d}$, which is annihilated by all $\psi_{a}^{\dagger}$. As basis in $\mathscr{C}_{d-p}$ we choose

$$
\left.\left.\| a_{1} \ldots a_{p}\right\rangle=\psi_{a_{1}} \ldots \psi_{a_{p}} \| 0\right\rangle
$$

such that an arbitrary wave function in $\mathscr{H}_{d-p}$ has the expansion

$$
\left.\Psi=f_{a_{1} \ldots a_{p}} \| a_{1} \ldots a_{p}\right\rangle, \quad f_{a_{1} \ldots a_{p}} \text { totally antisymmetric. }
$$

The supersymmetric Hamiltonian acts on such a state as follows:

$$
\langle\Psi \| H \Psi\rangle=\left(-\Delta+V^{(d)}\right) f_{a_{1} \ldots a_{p}}-2 \sum_{b, i=1}^{p}(-)^{i} \chi_{a_{i} b} f_{b a_{1} \ldots \check{a}_{i} \ldots a_{p}} .
$$

A comparison with (27) yields the important duality relation

$$
\left.H^{\chi}\right|_{\mathscr{H}_{p}}=\left.H^{-\chi}\right|_{\mathscr{H}_{d-p}},
$$

which states, that the Hamiltonians in $\mathscr{H}_{p}$ and $\mathscr{H}_{d-p}$ are equivalent, up to a signchange of the superpotential. Similarly on finds, that the action of $Q$ on the state (22) in $\mathscr{H}_{p}$ is identical to the action of $Q^{\dagger}$ on the state (29) in $\mathscr{H}_{d-p}$, up to a sign-change of $\chi$.

\section{The supersymmetric hydrogen atom and its symmetries}

First we consider $d$-dimensional supersymmetric systems with spherically symmetric superpotentials and derive the conserved angular momentum $J_{a b}$. The total angular momentum $J_{a b}$ is the sum of two terms: the orbital part $L_{a b}$ and the internal part $S_{a b}$ which transforms the components of a wave function. For a superpotential $\chi(r)$ the supercharges simplify to 


$$
Q=\mathrm{i} \psi_{a}\left(\partial_{a}+x_{a} f\right) \text { and } Q^{\dagger}=\mathrm{i} \psi_{a}^{\dagger}\left(\partial_{a}-x_{a} f\right), \quad \text { where } f=\frac{\chi^{\prime}}{r} .
$$

They should be scalars with respect to the rotation group $S O(d)$. However, since $\partial_{a}$ is a vector and $\psi_{a}$ a scalar with respect to $L_{a b}$, we need to supplement orbital rotations by internal ones such that $\psi_{a}$ becomes a vector as well. One easily verifies that

$$
\left[S_{a b}, \psi_{\mathrm{c}}\right]=\mathrm{i}\left(\delta_{a c} \psi_{b}-\delta_{b c} \psi_{a}\right), \quad \text { where } S_{a b}=\frac{1}{\mathrm{i}}\left(\psi_{a}^{\dagger} \psi_{b}-\psi_{b}^{\dagger} \psi_{a}\right),
$$

and that the hermitian $S_{a b}$ satisfy the same commutation relations (5) as the orbital angular momentum. It follows at once from (32) that the supercharge (31) commutes with the total angular momenta

$$
J_{a b}=L_{a b}+S_{a b},
$$

since $Q$ only contains scalar products of vectors operators.

Next we prove that there exists a supersymmetric generalization of the LaplaceRunge-Lenz vector in $d$ dimensions if the potential is $\sim 1 / r$. For such a potential the supercharges (31) do commute with the supersymmetric generalization of (6),

$$
C_{a}=J_{a b} p_{b}+p_{b} J_{a b}+x_{a} f(r) A,
$$

with a suitable operator $A$. One can show that $f$ must be the function in (31) for $C_{a}$ to commute with $Q$. This function, along with $A$ are fixed as follows:

First, for $C_{a}$ to be a vector, the operator $A$ must be a scalar under rotations induced by the $J_{a b}$. Second, in the zero-particle sector $\mathscr{H}_{0}$ the vector $C_{a}$ must coincide with $C_{a}$ in (6). Third, $A$ should commute with the particle number operator since the $J_{a b}$ do commute.

A general ansatz for $A$ subject to these three conditions reads

$$
A=\alpha \mathbb{1}-\beta N-\gamma S^{\dagger} S, \quad S=\hat{x}_{a} \psi_{a}, \quad \hat{x}_{a}=\frac{x_{a}}{r},
$$

with constants $\alpha, \beta$, and $\gamma$ which ought to be determined. Clearly, $C_{a}$ is a vector operator, such that

$$
\left[J_{a b}, C_{\mathrm{c}}\right]=\mathrm{i}\left(\delta_{a c} C_{b}-\delta_{b c} C_{a}\right)
$$

holds true. Let us now calculate the commutators between the $C_{a}$ and the supercharge. They should vanish for a suitable chosen function $f$ in (34). We obtain

$$
\begin{aligned}
{\left[C_{a}, Q\right]=} & 2\left\{f \psi_{b}+f^{\prime} S x_{b}\right\} J_{a b}+\beta f x_{a} Q_{0}+\mathrm{i} f x_{a}\left\{(\beta+\gamma) r f+\gamma \partial_{r}\right\} S \\
& +\mathrm{i}\left\{f \psi_{a}+f^{\prime} S x_{a}\right\}(1-d-A)+\mathrm{i} \gamma \hat{x}_{a} f(d-N-1) S .
\end{aligned}
$$

The terms containing derivatives are

$$
f x_{a}(\beta-2) Q_{0}+2 \mathrm{i} r\left(f+r f^{\prime}\right) S \partial_{a}+\mathrm{i}\left(\gamma f-2 r f^{\prime}\right) S x_{a} \partial_{r} .
$$

They cancel if

$$
f=-\frac{\lambda}{r} \quad \text { or } \quad \chi=-\lambda r \quad \text { and } \quad \beta=-\gamma=2
$$


hold true. With this choice all but the terms proportional to $S_{a b}$ in the first line on the right in (36) cancel, and we remain with

$$
\left[C_{a}, Q\right]=\frac{\mathrm{i} \lambda}{r}(\alpha-d+1)\left(\psi_{a}-\hat{x}_{a} S\right)
$$

which vanishes for $\alpha=d-1$. Hence, the supersymmetric extension of the conserved Laplace-Runge-Lenz vector reads

$$
C_{a}=J_{a b} p_{b}+p_{b} J_{a b}-\lambda \hat{x}_{a} A \quad \text { with } A=(d-1) 1-2 N+2 S^{\dagger} S,
$$

and this is the main result of this section. The choice $\chi=-\lambda r$ for the superpotential in (25) and (26) leads to the following supersymmetric extension of the Coulomb Hamiltonian:

$$
H=-\Delta+\lambda^{2}-\frac{\lambda A}{r} .
$$

Restricted to the zero-particle sector this is the Hamiltonian of the hydrogen atom 6 and restricted to the $d$ particle sector it corresponds to the electron-antiproton scattering system. The corresponding supercharge and its adjoint take the simple form

$$
Q=Q_{0}-\mathrm{i} \lambda S \text { and } Q^{\dagger}=Q_{0}^{\dagger}+\mathrm{i} \lambda S^{\dagger},
$$

where the free supercharge has been defined in (25) and the operator $S=\hat{x}_{a} \psi_{a}$ has already been used in (35).

\section{Algebraic determination of the spectrum}

We proceed as we did in the purely bosonic case and calculate the commutator of two $C_{a}$ :

$$
\left[C_{a}, C_{b}\right]=-4 \mathrm{i} J_{a b}\left(-\Delta-\frac{\lambda}{r} A\right) \stackrel{(39)}{=}-4 \mathrm{i} J_{a b}\left(H-\lambda^{2}\right) .
$$

Up to the shift in $H$ and the replacement $L_{a b} \rightarrow J_{a b}$ this is the same relation as (7). The total angular momenta $J_{a b}$ and the vector operator

$$
K_{a}=\frac{C_{a}}{\sqrt{4\left(\lambda^{2}-H\right)}}
$$

form a $S O(d+1)$ algebra on the subspace of bound states $\left(E<\lambda^{2}\right)$. Finally we should calculate $C_{a} C_{a}$. If we can express this scalar operator in terms of $H$ and operators that commute with $H$, similarly as we did in (10), then we may solve for $H$. However, one soon realizes that this is impossible by only using the operators $1, N$,

\footnotetext{
${ }^{6}$ We have to identify $\eta=\lambda(d-1)$. The additional shift $\lambda^{2}$ makes the lowest eigenvalue of this operator to be equal to 0 .
} 
$J_{a b} J_{a b}$, and $H$. However, we can express $C_{a} C_{a}$ in terms of $1, N, J_{a b} J_{a b}$ and the two operators $Q Q^{\dagger}$ and $Q^{\dagger} Q$ as follows:

$$
\begin{aligned}
C_{a} C_{a}= & 4\left(\lambda^{2}-H\right) K_{a} K_{a} \\
= & -2 \lambda^{2} J_{a b} J_{a b}+\left(2 J_{a b} J_{a b}+(d-2 N-1)^{2}\right) Q Q^{\dagger} \\
& +\left(2 J_{a b} J_{a b}+(d-2 N+1)^{2}\right) Q^{\dagger} Q,
\end{aligned}
$$

and this relation is sufficient to obtain the spectrum of the supersymmetric hydrogen atom.

Each of the three subspaces in the decomposition (28) is left invariant by the Hamiltonian and we may consider $H$ on each subspace separately. Since $Q^{2}=0$ we find $\left.H\right|_{Q \mathscr{H}}=Q Q^{\dagger}$ and $\left.H\right|_{Q^{\dagger} \mathscr{H}}=Q^{\dagger} Q$ and can solve (41) for $H$ in each of these subspaces

$$
\begin{aligned}
& \left.H\right|_{Q \mathscr{H}}=Q Q^{\dagger}=\lambda^{2}-\frac{(d-2 N-1)^{2} \lambda^{2}}{(d-2 N-1)^{2}+4 \mathscr{C}_{(2)}}, \\
& \left.H\right|_{Q^{\dagger} \mathscr{H}}=Q^{\dagger} Q=\lambda^{2}-\frac{(d-2 N+1)^{2} \lambda^{2}}{(d-2 N+1)^{2}+4 \mathscr{C}_{(2)}},
\end{aligned}
$$

where $\mathscr{C}_{(2)}$ is the second-order Casimir of the dynamical symmetry group $S O(d+1)$,

$$
\mathscr{C}_{(2)}=\frac{1}{2} J_{A B} J_{A B}=\frac{1}{2} J_{a b} J_{a b}+K_{a} K_{a} .
$$

All zero modes of $H$ are annihilated by both $Q$ and $Q^{\dagger}$, and according to (41) the second-order Casimir must vanish on these modes, such that

$$
\left.\mathscr{C}_{(2)}\right|_{\operatorname{Ker} H}=0 \text {. }
$$

We conclude that every normalizable zero-mode $\Psi$ of $H$ must transform trivially under the dynamical symmetry group, $J_{A B} \Psi=0$.

To obtain the bound state energies we need to determine those irreducible representations of the dynamical symmetry group which are realized in $\mathscr{H}$ and the corresponding values of the second-order Casimir operator. The degeneracy of an energy level is then equal to the dimension of the corresponding representation.

We use the abbreviation $\mathscr{D}_{\wp}^{\ell}$ to denote multiplets of the orthogonal groups corresponding to Young tableaux of the form

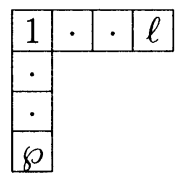

since in the following only those representations will appear. Let us assume, that each component function $f_{a_{1} \ldots a_{p}}(x)$, entering the state $\Psi \in \mathscr{H}_{p}$ in (24), is a harmonic polynomial of degree $\ell$, i.e., 


$$
f_{a_{1} \ldots a_{p}}(x)=\sum_{b_{1}, b_{2}, \ldots, b_{\ell}} f_{a_{1} \ldots a_{p} b_{1} \ldots b_{\ell}} x_{b_{1}} x_{b_{2}} \ldots x_{b_{\ell}},
$$

with $f$ symmetric in the $b$-indices and of zero trace in each pair of them. Since $f_{a_{1} \ldots a_{p}}$ is completely antisymmetric in the $a$-indices, these objects transform according to the totally antisymmetric representation

$$
\mathcal{D}_{\wp}^{1} \sim \begin{array}{|l|}
\hline 1 \\
\hline \cdot \\
\hline \varnothing \\
\hline
\end{array}
$$

of the rotation group $S O(d)$ generated by the $S_{a b}$. On the other hand, each homogeneous polynomial $f_{a_{1} \ldots a_{p}}(x)$ transforms according to the totally symmetric representations

$$
\mathcal{D}_{1}^{\ell} \sim \begin{array}{|l|l|l|l|}
\hline 1 & \cdot & \cdot & \ell \\
\hline
\end{array}
$$

of the rotation group generated by the $L_{a b}$. It follows that the wave function $\Psi \in \mathscr{H}_{p}$ transforms according to the tensor-product representations

$$
\mathscr{D}_{p}^{1} \otimes \mathscr{D}_{1}^{\ell}=\mathscr{D}_{p-1}^{\ell} \oplus \mathscr{D}_{p}^{\ell-1} \oplus \mathscr{D}_{p}^{\ell+1} \oplus \mathscr{D}_{p+1}^{\ell} \text {. }
$$

Recall that $p$ is the fermion number and $\ell$ the order of the homogeneous polynomials. For $\psi \in \mathscr{H}_{0}$ or $\psi \in \mathscr{H}_{d}$ the first factor on the left-hand side in (44) becomes the trivial representation and we only obtain the fully symmetric representations $\mathscr{D}_{1}^{\ell}$ on the right-hand side, in agreement with our earlier results in the purely bosonic case. In the sectors $\mathscr{H}_{1}$ and $\mathscr{H}_{d-1}$ the first representation on the right-hand side of (44) is absent. For linear functions with $\ell=1$ the second representation on the right is missing. Finally, when using the results (44), one should keep in mind that the representations with $\wp$ and $d-\wp$ of $S O(d)$ are equivalent, $\mathscr{D}_{\wp}^{\ell} \sim \mathscr{D}_{d-\wp}^{\ell}$, and that for even dimensions the representations $\mathscr{D}_{d / 2}^{\ell}$ are reducible and contain one selfdual and one anti-selfdual multiplet. All representations of the rotation group $S O(d)$ appearing in the tensor product (44) should group together into multiplets of the dynamical symmetry group $S O(d+1)$. To continue we need the following rules for the branching of $S O(d+1)$ - into $S O(d)$-representations: ${ }^{7}$

$$
\left.\left.\mathscr{D}_{\wp}^{\ell}\right|_{S O(d+1)} \rightarrow\left\{\mathscr{D}_{\wp}^{\ell} \oplus \mathscr{D}_{\wp}^{\ell-1} \oplus \cdots \oplus \mathscr{D}_{\wp}^{1} \oplus \mathscr{D}_{\wp-1}^{\ell} \oplus \mathscr{D}_{\wp-1}^{\ell-1} \oplus \cdots \oplus \mathscr{D}_{\wp-1}^{1}\right\}\right|_{S O(d)} .
$$

Now it is not difficult to see that in the sector $\mathscr{H}_{p}$ all polynomials up to order $\ell$ appear in the branching of only two $S O(d+1)$-multiplets up to two extreme representations

\footnotetext{
${ }^{7}$ They can be obtained from [14] or by using the program LiE.
} 


$$
\left.\left.\mathscr{D}_{\wp}^{\ell} \oplus \mathscr{D}_{\wp+1}^{\ell}\right|_{S O(d+1)} \rightarrow\left\{\mathscr{D}_{\wp}^{1} \otimes\left(1 \oplus \mathscr{D}_{1}^{1} \oplus \cdots \oplus \mathscr{D}_{1}^{\ell}\right)-\mathscr{D}_{\wp}^{\ell+1}+\mathscr{D}_{\wp-1}^{\ell}\right\}\right|_{S O(d)} .
$$

Of course, for $\wp=1$ the last representation of the rotation group is absent. There is one notable exception to these branching rules for even $d$ : in the middle sector $\mathscr{H}_{n=d / 2}$ the correct branching rule reads

$$
\left.\left.\mathscr{D}_{n}^{\ell} \oplus \mathscr{D}_{n}^{\ell}\right|_{S O(d+1)} \rightarrow\left\{\mathscr{D}_{n}^{1} \otimes\left(1 \oplus \mathscr{D}_{1}^{1} \oplus \cdots \oplus \mathscr{D}_{1}^{\ell}\right)-\mathscr{D}_{n}^{\ell+1}-\mathscr{D}_{n}^{\ell}-\mathscr{D}_{n-1}^{\ell}\right\}\right|_{S O(d)} .
$$

We summarize our results: In odd dimensions $d=2 n+1$ the following representations of $S O(2 n+2)$ containing bound states arise in the various sectors of $\mathscr{H}$ for $\ell \geqslant 1$ :

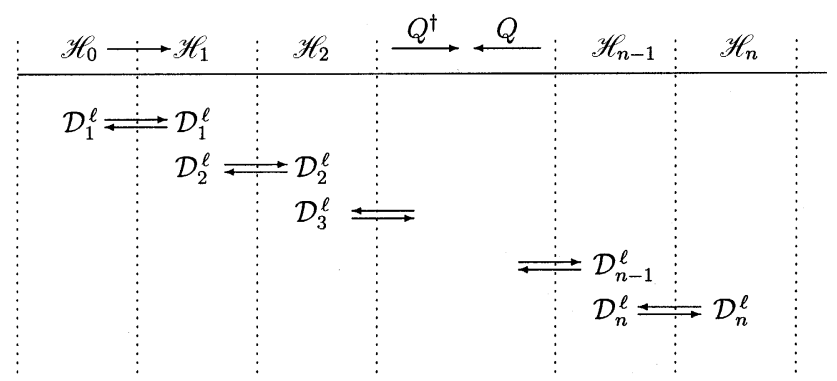

In all sectors but $\mathscr{H}_{0}$ we have $\ell \in \mathbb{N}$. In the subspaces $\mathscr{H}_{0}$ we have $\ell \in \mathbb{N}_{0}$ and $\ell=0$ corresponds to the trivial representation. The sectors $\mathscr{H}_{p>n}$ support no bound states (see below) and therefore it suffices to consider the sectors with $N \leqslant n$.

In even dimensions $d=2 n$ the following representations of the dynamical symmetry group $S O(2 n+1)$ arise for $\ell \geqslant 1$ :

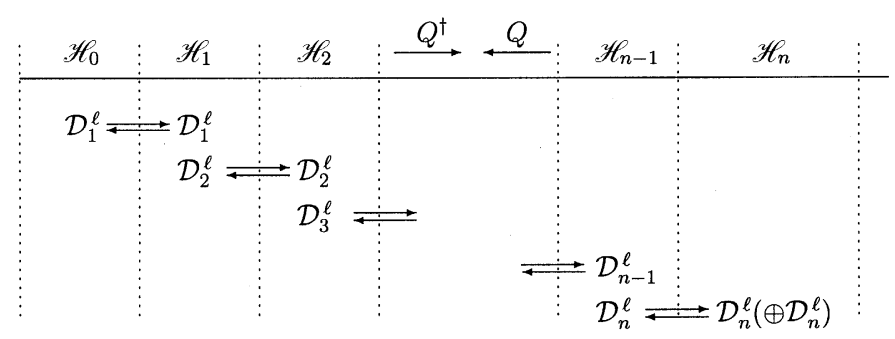

The values of the quadratic Casimir operator of $S O(d+1)$ entering the formulae (42), (43) for the energies are

$$
\mathscr{C}_{(2)}\left(\mathscr{D}_{\wp}^{\ell}\right)=d(\ell+\wp-1)+\ell(\ell-1)-\wp(\wp-1) .
$$

The dimensions of the representations $\mathscr{D}_{\phi}^{\ell}$ are given in the appendix. In odd dimensions one uses the formula (A.8) with $2 n$ replaced by $2 n=d+1$ and in even dimensions the formula (A.17) with $d+1=2 n+1$. In addition one must set 


$$
\left(\ell_{1}, \ell_{2}, \ldots, \ell_{n}\right)=(\overbrace{\ell, 1, \ldots, 1}^{p}, 0, \ldots, 0)
$$

in these formulae.

Now we are ready to determine all eigenvalues of the supersymmetric Hamiltonian (39) with the help of the results (42), (43), and (46) as follows.

In $\mathscr{H}_{0}$ only the symmetric representations $\mathscr{D}_{1}^{\ell}$ of $S O(d+1)$ are realized. Since in addition $\left.Q\right|_{\mathscr{H}_{0}}=0$ we obtain the following eigenvalues for $H$ in (42):

$$
\mathscr{H}_{0}: E_{0}\left(\mathscr{D}_{1}^{\ell}\right)=\lambda^{2}-\left(\frac{d-1}{d-1+2 \ell}\right)^{2} \lambda^{2}, \quad \ell \in \mathbb{N}_{0} .
$$

The index 0 at the energy $E$ indicates the zero-particle sector. Since the supercharges commute with the dynamical symmetry group the multiplet $\mathscr{D}_{1}^{\ell}$ is paired with the same multiplet in $\mathscr{H}_{1}$. The eigenfunctions in $\mathscr{H}_{1}$ are obtained by acting with $Q^{\dagger}$ on those in $\mathscr{H}_{0}$. According to our previous results (see the figure above) there exists the additional multiplet $\mathscr{D}_{2}^{\ell}$ in $\mathscr{H}_{1}$. This is obtained by acting with $Q$ on the same representation in the two-particle sector. Hence $H=Q Q^{\dagger}$ on this second multiplet and we obtain

$$
\begin{aligned}
& E_{1}\left(\mathscr{D}_{1}^{\ell}\right)=\lambda^{2}-\left(\frac{d-1}{d-1+2 \ell}\right)^{2} \lambda^{2}, \quad \ell \in \mathbb{N}, \\
& E_{1}\left(\mathscr{D}_{2}^{\ell}\right)=\lambda^{2}-\left(\frac{d-3}{d-1+2 \ell}\right)^{2} \lambda^{2}, \quad \ell \in \mathbb{N} .
\end{aligned}
$$

Note that $\ell=0$ does not occur in $\mathscr{H}_{1}$. In $\mathscr{H}_{0}$ the states with $\ell=0$ have vanishing energy and hence are annihilated by $Q^{\dagger}$.

Now one continues with $\mathscr{H}_{2}$ and further on to $\mathscr{H}_{3}$ etc. One only needs the formulae

$$
\begin{aligned}
& E_{p}\left(\mathscr{D}_{p}^{\ell}\right)=\left.Q^{\dagger} Q\right|_{\mathscr{H}_{p}}\left(\mathscr{D}_{p}^{\ell}\right)=\lambda^{2}-\left(\frac{d+1-2 p}{d-1+2 \ell}\right)^{2} \lambda^{2}, \quad \ell \in \mathbb{N}, \\
& E_{p}\left(\mathscr{D}_{p+1}^{\ell}\right)=\left.Q Q^{\dagger}\right|_{\mathscr{H}_{p}}\left(\mathscr{D}_{p+1}^{\ell}\right)=\lambda^{2}-\left(\frac{d-1-2 p}{d-1+2 \ell}\right)^{2} \lambda^{2}, \quad \ell \in \mathbb{N} .
\end{aligned}
$$

We shall determine the corresponding eigenfunctions in the following section.

\section{Eigenstates of the supersymmetric hydrogen atom}

So far we have not considered which highest weight states of the dynamical symmetry group are normalizable. Now we explicitly construct these states in all subspaces $\mathscr{H}_{p} \subset \mathscr{H}$. In the previous section we have seen that for any $\ell \geqslant 1$ there are one or two irreducible representations of $S O(d+1)$, namely

$$
\mathscr{D}_{p}^{\ell} \subset\left(\mathscr{H}_{p} \cap Q^{\dagger} \mathscr{H}\right) \text { and } \quad \mathscr{D}_{p+1}^{\ell} \subset\left(\mathscr{H}_{p} \cap Q \mathscr{H}\right) \text {. }
$$


It suffices to construct the highest weight states $\Psi_{p}\left(\mathscr{D}_{p+1}^{\ell}\right)$ of the latter multiplets. The highest weight states $\Psi_{p}\left(\mathscr{D}_{p}^{\ell}\right)$ of the first set of multiplets in (47) are then just their superpartners

$$
\Psi_{p}\left(\mathscr{D}_{p}^{\ell}\right)=Q^{\dagger} \Psi_{p-1}\left(\mathscr{D}_{p}^{\ell}\right) .
$$

Actually we only need to calculate the highest weight states $\Psi_{p}\left(\mathscr{D}_{p+1}^{\ell}\right)$ for $p<d / 2$ because of the duality relation

$$
(p, \lambda) \leftrightarrow(d-p,-\lambda),
$$

which follows from (30) and (37).

Observe that for any normalizable $H$-eigenstate $\Psi \in Q \mathscr{H}$ the transformed state $Q^{\dagger} \Psi$ is normalizable, as can be seen from

$$
\left(Q^{\dagger} \Psi, Q^{\dagger} \Psi\right)=\left(\Psi, Q Q^{\dagger} \Psi\right)=(\Psi, H \Psi)=E(\Psi, \Psi) .
$$

Without calculating the highest weight states we can argue in which sectors bound states cannot exist. For that purpose we consider the Hamiltonian (39). It is easy to see that the hermitian operator $S^{\dagger} S$, where $S$ has been defined in (35), is an orthogonal projector, and hence has eigenvalues 0 and 1 . It follows at once that for $p>d / 2$ the operator $A$ in (38) is negative and hence $H>\lambda^{2}$. We conclude that $H$ has no bound states in the sectors $\mathscr{H}_{p>d / 2}$. On the particular sector $\mathscr{H}_{n}$ the operator $A$ has both positive and negative eigenvalues. We expect that in this sector only one of the two representations (for each $\ell$ ) of the dynamical symmetry group contains bound states.

After these general considerations we proceed with computing the highest weight states $\Psi_{p}\left(\mathscr{D}_{p+1}^{\ell}\right)$ in the subspace $\mathscr{H}_{p} \cap Q \mathscr{H}$. Again we proceed differently in even- and odd-dimensional spaces.

Even-dimensional spaces: We use the complex coordinates $z_{1}, \ldots, z_{n}$ in $\mathbb{R}^{d=2 n}$ and the creation/annihilation operators $\phi_{1}^{\dagger}, \ldots, \phi_{n}^{\dagger}, \phi_{1}, \ldots, \phi_{n}$ introduced in Appendix A.1, together with the complex conjugated objects. Since the dynamical symmetry group is $S O(2 n+1)$, we should take the Cartan operators $H_{i}$ and raising operators $E_{i}$ from Appendix A.2. However, we must remember that the last row and last column of $\left(J_{A B}\right)$ contain the components of the generalized Laplace-Runge-Lenz vector. As a consequence the last step operator $E_{n}$ in (A.16) is to be replaced by

$$
\begin{aligned}
E_{n}= & \frac{1}{\sqrt{2}}\left(K_{d-1}+\mathrm{i} K_{d}\right) \sim \frac{1}{\sqrt{2}}\left(C_{d-1}+\mathrm{i} C_{d}\right) \\
= & -2 z_{n} \Delta+\left(2 r \partial_{r}+d-1\right) \bar{\partial}_{n}-2 \phi_{n}^{\dagger}\left(\phi_{i} \bar{\partial}_{i}+\bar{\phi}_{i} \partial_{i}\right) \\
& +2\left(\phi_{i}^{\dagger} \partial_{i}+\bar{\phi}_{i}^{\dagger} \bar{\partial}_{i}\right) \bar{\phi}_{n}-\frac{\lambda z_{n}}{r} A .
\end{aligned}
$$

Since the simple roots of the rotational subgroup $S O(d)$ are positive roots of the dynamical symmetry group, a highest weight state of $S O(d+1)$ is automatically a highest weight state of $S O(d)$, similar as in the purely bosonic case. Since the two groups share the same Cartan generators the highest weight state $\Psi_{p}\left(\mathscr{D}_{p+1}^{\ell}\right)$ of $S O(d+1)$ must also be a highest weight state of the multiplet $\mathscr{D}_{p+1}^{\ell}$ of $S O(d)$. From the branching rule (45) and the tensor products (44) it follows that this highest 
weight state must be the state $\mathscr{Y}_{a}(\ell, p+1)$ given in (A.13). Hence we are lead to the ansatz

$$
\Psi_{p}\left(\mathscr{D}_{p+1}^{\ell}\right)=f(r) \mathscr{Y}_{a}(\ell, p+1) .
$$

It remains to determine the radial function $f(r)$ such that $\Psi_{p}$ is annihilated by $E_{n}$. With $E_{n}$ from (48) one finds the following equation for the radial function $f$ :

$$
(d-1+2 \ell) f^{\prime}+\lambda(d-1-2 p) f=0
$$

such that the relevant highest weight states in the $p$-particle sector take the form

$$
\Psi_{p}\left(\mathscr{D}_{p+1}^{\ell}\right)=\exp \left(-\gamma_{\ell p} r\right) \mathscr{Y}_{a}(\ell, p+1) \quad \text { with } \gamma_{\ell p}=\frac{d-1-2 p}{d-1+2 \ell} \lambda .
$$

As $\lambda$ is assumed positive, these are bound states for $p<n$.

Odd-dimensional spaces: For odd dimensions $d=2 n+1$ the rank of the dynamical symmetry group $S O(2 n+2)$ exceeds the rank of the rotation group $S O(d)$ by 1 . As in the purely bosonic case we combine the first $2 n$ coordinates, creation and annihilation operators to complex ones (cf. Appendix A.2). Since the rank of the dynamical symmetry group is even, we should take the Cartan generators from Appendix A. 1 with $n$ replaced by $n+1$. Since $\left(J_{A B}\right)$ contains the Laplace-RungeLenz vector the explicit realization of these operators differs from the one in this appendix. More precisely, the first $n$ Cartan generators are those in (A.4), but the last one $H_{n+1}$ is $K_{d} \sim C_{d}$ (cf. Appendix B), where

$$
C_{d}=-2 x_{d} \Delta+\left(2 r \partial_{r}+d-1\right) \partial_{d}-2 \psi_{d}^{\dagger}\left(\phi_{i} \bar{\partial}_{i}+\bar{\phi}_{i} \partial_{i}\right)+2\left(\phi_{i}^{\dagger} \partial_{i}+\bar{\phi}_{i}^{\dagger} \bar{\partial}_{i}\right) \psi_{d}-\lambda \hat{x}_{d} A .
$$

The raising operators are the $n+1$ operators $E_{i}$ in (A.6) plus the two operators ${ }^{8}$

$$
E_{n}=\frac{1}{\mathrm{i}}\left(z_{n} \partial_{x_{d}}-x_{d} \bar{\partial}_{z_{n}}+\phi_{n}^{\dagger} \psi_{d}-\psi_{d}^{\dagger} \bar{\phi}_{n}\right) \quad \text { and } \quad E_{n+1}=\frac{1}{\sqrt{2}}\left(C_{d-2}+\mathrm{i} C_{d-1}\right) .
$$

The last operator coincides with $E_{n}$ in (48). By using similar arguments as in even dimensions we are led to the following ansatz:

$$
\Psi_{p}\left(\mathscr{D}_{p+1}^{\ell}\right)=f(r) \mathscr{Y}_{a}(\ell, p+1)
$$

for the highest weight state of $\mathscr{D}_{p+1}^{\ell} \subset \mathscr{H}_{p}$. This function is annihilated by all $E_{i \leqslant n}$. The condition $E_{n+1} \Psi_{p}=0$ yields the same differential equation for the radial function $f$ as before and we obtain

$$
\Psi_{p}\left(\mathscr{D}_{p+1}^{\ell}\right)=\exp \left(-\gamma_{\ell p} r\right) \mathscr{Y}_{a}(\ell, p+1) \quad \text { with } \gamma_{\ell p}=\frac{d-1-2 p}{d-1+2 \ell} \lambda .
$$

For positive $\lambda$ these states are normalizable for all $p<n$. It is easy to see that the last Cartan generator $\sim C_{d}$ annihilates this state and this shows that it has the correct weight.

\footnotetext{
${ }^{8}$ Which are independent combinations of the last two raising operators, see Appendix B.
} 
The remaining highest weight states: We have argued that the highest weight state $\Psi_{p+1}\left(\mathscr{D}_{p+1}^{\ell}\right) \subset \mathscr{H}_{p+1}$ is the superpartner of $\Psi_{p}\left(\mathscr{D}_{p+1}^{\ell}\right)$ in (52). A simple calculation yields

$$
\begin{aligned}
\Psi_{p+1}\left(\mathscr{D}_{p+1}^{\ell}\right) & =Q^{\dagger} \Psi_{p}\left(\mathscr{D}_{p+1}^{\ell}\right) \\
& =\mathrm{i}\left(\left(\lambda-\gamma_{\ell p}\right) S^{\dagger} \mathscr{Y}_{a}(\ell, p+1)+(\ell+p) \mathscr{Y}_{s}(\ell, p+1)\right) \exp \left(-\gamma_{\ell p} r\right)
\end{aligned}
$$

for this state and shows that it is a linear combination of the two highest weight states $\mathscr{Y}_{s}$ and $\mathscr{Y}_{a}$ of $S O(d)$ given in formulae (A.12) and (A.13). These states lead to additional bound-state multiplets in the sectors $\mathscr{H}_{p}$ with $p=1, \ldots, n$.

\section{The supersymmetric hydrogen atom in dimensions $\leqslant 4$}

In this section we apply the general results of the previous three sections to supersymmetric systems in low dimensions. The two-dimensional case mainly serves as illustration of the method. It may be worth noting that such systems admit a supersymmetric Laplace-Runge-Lenz vector, contrary to what has been claimed in the literature [15]. The three-dimensional quantum system is of course the most interesting extension of the ordinary hydrogen atom. We have included the four-dimensional supersymmetric system since it already shows very nicely which additional structures arise when one goes beyond three dimensions.

Two dimensions: The Hilbert space of the supersymmetric hydrogen atom in two space dimensions contains three sectors

$$
\mathscr{H}=\mathscr{H}_{0} \oplus \mathscr{H}_{1} \oplus \mathscr{H}_{2},
$$

and an arbitrary wave function has the expansion (in the basis (22))

$$
\Psi=f_{0}|0\rangle+\left(f_{1}|1\rangle+f_{2}|2\rangle\right)+f_{12}|12\rangle \sim f=\left(f_{0}, f_{1}, f_{2}, f_{12}\right)^{\mathrm{T}} .
$$

The Hamiltonian (39) acts on the component functions in $f$ as follows:

$$
f \mapsto H f \quad \text { with } H=-\Delta+\lambda^{2}+\frac{\lambda}{r}\left(\begin{array}{lll}
-1 & 0 & 0 \\
0 & \delta_{a b}-2 \hat{x}_{a} \hat{x}_{b} & 0 \\
0 & 0 & 1
\end{array}\right) .
$$

Clearly, for $\lambda>0$ there are no bound states in the two-particle subspace, in accordance with our general result below Eq. (47). Only the multiplets

$$
\mathscr{D}_{1}^{\ell \geqslant 0} \subset \mathscr{H}_{0} \text { and } \mathscr{D}_{1}^{\ell>0} \subset \mathscr{H}_{1}
$$

of the dynamical symmetry group $S O(3)$ contain normalizable states.

We introduce the complex coordinate $z$ and the complex creation/annihilation operator (see Appendix A.1) in terms of which the highest weight state (49) read

$$
\Psi_{0}\left(\mathscr{D}_{1}^{\ell}\right)=\exp \left(-\gamma_{\ell 0} r\right) \mathscr{Y}_{a}(\ell, 1)=\exp \left(-\gamma_{\ell 0} r\right) z^{\ell}|0\rangle, \quad \gamma_{\ell 0}=\frac{\lambda}{1+2 \ell},
$$

and its superpartner (53)

$$
\Psi_{1}\left(\mathscr{D}_{1}^{\ell}\right)=Q^{\dagger} \Psi_{0}\left(\mathscr{D}_{1}^{\ell}\right)=\mathrm{i}\left(\left(\lambda-\gamma_{\ell 0}\right) S^{\dagger} \mathscr{Y}_{a}(\ell, 1)+\ell \mathscr{Y}_{s}(\ell, 1)\right) \exp \left(-\gamma_{\ell 0} r\right) .
$$


The energy of the $\ell+1$ states in each of the two corresponding $S O(3)$-multiplets is

$$
E_{\ell}=\lambda^{2}-\gamma_{\ell 0}^{2} \text {. }
$$

There is exactly one zero-energy ground state in the zero-particle sector and this state has $\ell=0$. It is annihilated by the adjoint supercharge $Q^{\dagger}$, as can be seen from (54). The spectrum of the supersymmetric system is depicted in the following figure.

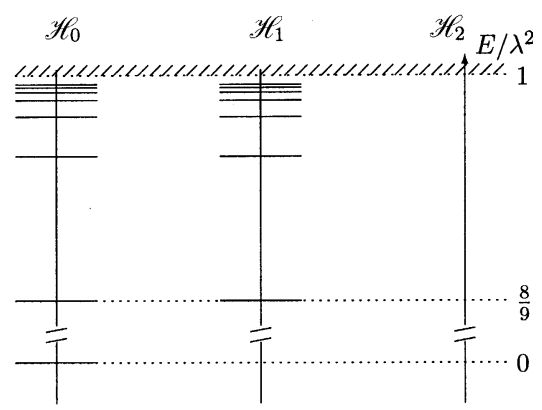

Eigenvalues of $H$ in $d=2$ dimensions.

Three dimensions: For this most relevant system, the Hilbert space contains four sectors

$$
\mathscr{H}=\mathscr{H}_{0} \oplus \mathscr{H}_{1} \oplus \mathscr{H}_{2} \oplus \mathscr{H}_{3},
$$

and a wave function has the expansion

$$
\Psi=f_{0}|0\rangle+\left(f_{1}|1\rangle+f_{2}|2\rangle+f_{3}|3\rangle\right)+\cdots
$$

Since here we are only interested in bound states it suffices to consider $H$ on the subsectors $\mathscr{H}_{0}$ and $\mathscr{H}_{1}$. The Hamiltonian in $\mathscr{H}_{0}$ belongs to the ordinary hydrogen atom

$$
H^{(0)}=-\Delta+\lambda^{2}-\frac{2 \lambda}{r}
$$

and it acts on a state $\Psi \in \mathscr{H}_{1}$ with component functions $\langle a \mid H\rangle=f_{a}$ as follows:

$$
\langle a \mid H \Psi\rangle=\left(-\Delta+\lambda^{2}\right) f_{a}-\frac{2 \lambda}{r} \hat{x}_{a} \hat{x}_{b} f_{b} .
$$

We take the coordinates $\left(z, x_{3}\right)$ and the creation operators $\left(\phi^{\dagger}, \bar{\phi}^{\dagger}, \psi_{3}^{\dagger}\right)$ (see Appendix A.2). The highest weight state in the multiplet $\mathscr{D}_{1}^{\ell} \subset \mathscr{H}_{0}$ of the dynamical symmetry group $S O(4)$ has the form

$$
\Psi_{0}\left(\mathscr{D}_{1}^{\ell}\right)=\exp \left(-\gamma_{\ell 0} r\right) \mathscr{Y}_{a}(\ell, 1) \in \mathscr{H}_{0}, \quad \gamma_{\ell 0}=\frac{\lambda}{1+\ell},
$$

and this state is mapped into the partner state

$$
\Psi_{1}\left(\mathscr{D}_{1}^{\ell}\right)=Q^{\dagger} \Psi_{0}\left(\mathscr{D}_{1}^{\ell}\right)=\mathrm{i}\left(\left(\lambda-\gamma_{\ell 0}\right) S^{\dagger} \mathscr{Y}_{a}(\ell, 1)+\ell \mathscr{Y}_{s}(\ell, 1)\right) \exp \left(-\gamma_{\ell 0} r\right) .
$$

All states in the two corresponding $S O(4)$-multiplets share the same energy

$$
E_{\ell}=\lambda^{2}-\gamma_{\ell 0}^{2}
$$


and both multiplets contain $(\ell+1)^{2}$ states. This is the well-known spectrum of the hydrogen atom. The normalizable zero-mode has $\ell=0$ and resides in the zeroparticle sector. It is just the ground state of the hydrogen atom.

There are no further bound states, since the other states are paired with wave functions in $\mathscr{H}_{2}$ and thus cannot be normalizable. The spectrum of the supersymmetric hydrogen atom in three dimensions is shown below:

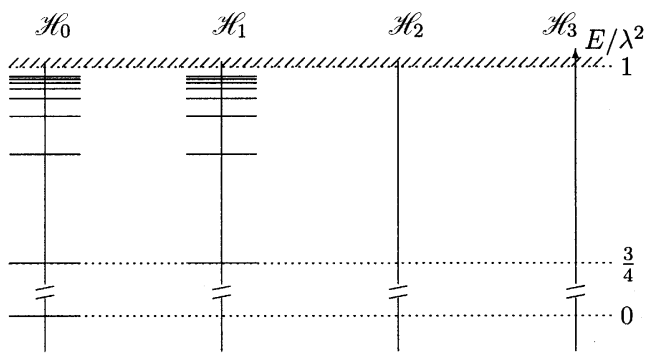

Eigenvalues of $H$ in $d=3$ dimensions.

Four dimensions: The Hilbert space splits into five subsectors

$$
\mathscr{H}=\mathscr{H}_{0} \oplus \mathscr{H}_{1} \oplus \mathscr{H}_{2} \oplus \mathscr{H}_{3} \oplus \mathscr{H}_{4} .
$$

In the zero-particle sector the Hamiltonian takes the form

$$
H^{(0)}=-\Delta+\lambda^{2}-\frac{3 \lambda}{r}
$$

and in the one-particle sector it acts on a state $\Psi$ with $\langle a \mid \Psi\rangle=f_{a}$ as follows:

$$
\langle a \mid H \Psi\rangle=\left(-\Delta+\lambda^{2}-\frac{\lambda}{r}\right) f_{a}-\frac{2 \lambda}{r} \hat{x}_{a} \hat{x}_{b} f_{b} .
$$

In $\mathscr{H}_{2}$ we are left with a $6 \times 6$-matrix Schrödinger operator: For a two-particle state $\Psi$ with component function $\langle a b \mid \Psi\rangle=f_{a b}$ we obtain

$$
\langle a b \mid H \Psi\rangle=\left(-\Delta+\lambda^{2}+\frac{\lambda}{r}\right) f_{a b}-\frac{2 \lambda}{r}\left(\hat{x}_{b} f_{a c}-\hat{x}_{a} f_{b c}\right) \hat{x}_{\mathrm{c}} .
$$

We introduce complex coordinates $z_{1}, z_{2}$ and creation operators $\phi_{1}^{\dagger}, \phi_{2}^{\dagger}$ as in Appendix A.1.

For all $\ell \in \mathbb{N}$ we find the highest weight states

$$
\Psi_{0}\left(\mathscr{D}_{1}^{\ell}\right)=\exp \left(-\gamma_{\ell 0} r\right) z_{1}^{\ell}|0\rangle \quad \text { with } \gamma_{\ell 0}=\frac{3 \lambda}{2 \ell+3}
$$

in the zero-particle sector, together with their superpartners in $\mathscr{H}_{1}$,

$$
\Psi_{1}\left(\mathscr{D}_{1}^{\ell}\right)=\mathrm{i}\left(\left(\lambda-\gamma_{\ell 0}\right) S^{\dagger} \mathscr{Y}_{a}(\ell, 1)+\ell \mathscr{Y}_{s}(\ell, 1)\right) \exp \left(-\gamma_{\ell 0} r\right),
$$

which exists for $\ell>0$. All states in these two multiplets have the same energy

$$
E_{\ell}=\lambda^{2}-\gamma_{\ell 0}^{2},
$$


and the number of states in each multiplet is $(\ell+1)(\ell+2)(2 \ell+3) / 6$. Now there is an additional representation $\mathscr{D}_{2}^{\ell} \subset \mathscr{H}_{1}$ with highest weight state

$$
\begin{aligned}
& \Psi_{1}\left(\mathscr{D}_{2}^{\ell}\right)=\exp \left(-\gamma_{\ell 1} r\right) \mathscr{Y}_{a}(\ell, 2)=\exp \left(-\gamma_{\ell 1} r\right)\left(\phi_{2}^{\dagger} z_{1}-\phi_{1}^{\dagger} z_{2}\right) z_{1}^{\ell-1}|0\rangle, \\
& \gamma_{\ell 1}=\frac{\lambda}{3+2 \ell} .
\end{aligned}
$$

The energy of this state is

$$
E_{\ell}=\lambda^{2}-\gamma_{\ell 1}^{2},
$$

and the multiplet contains $\ell(\ell+3)(2 \ell+3) / 4$ members. Again there is a supersymmetric partner multiplet with the same energy and degeneracy. The remaining highest weight state of $\mathscr{D}_{3}^{\ell} \simeq \mathscr{D}_{2}^{\ell} \subset \mathscr{H}_{2}$ is paired to a state in $\mathscr{H}_{3}$ and therefore not normalizable. Thus we find the spectrum as depicted in the following figure:

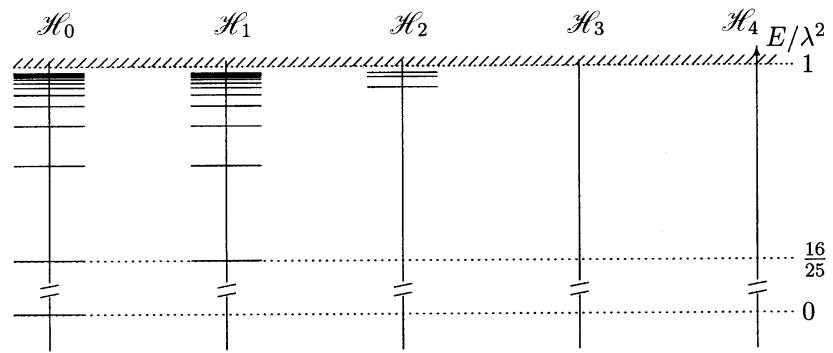

Eigenvalues of $H$ in $d=4$ dimensions.

\section{Summary and discussion}

We have succeeded in extending the celebrated results of Pauli, Fock, Bargmann and others in two directions: to higher dimensions and to the $\mathscr{N}=2$ supersymmetric extension of the hydrogen atom. First we constructed a generalized angular momentum and an extended Laplace-Runge-Lenz vector which could be combined to generators of the dynamical symmetry group $S O(d+1)$ in $d$ dimensions. Then we related the quadratic Casimir operator of this group to the particle number $N$, $Q Q^{\dagger}$ and $Q Q^{\dagger}$. This way we calculated the bound state spectrum of the supersymmetric hydrogen atom in arbitrary dimensions by algebraic means. We have determined all bound-state multiplets of the dynamical symmetry group and calculated the highest weight state in each of them.

Bander and Itzykson [7] distinguished between the infinitesimal and the global method to solve the Coulomb problem. The former is based on the LaplaceRunge-Lenz vector and is the method used in this paper. In the second method one performs a stereographic projection of the $d$-dimensional momentum space to the unit sphere in $d+1$ dimensions which in turn implies a $S O(d+1)$ symmetry group. It would be interesting to perform a similar global construction for the supersymmetric systems introduced in this paper. 
We have not explained why every multiplet of the dynamical symmetry group appears four times. Furthermore there is a new 'accidental' degeneracy: in higher dimensions some eigenvalues of the Hamiltonian appear in many different particle-number sectors. It may very well be that the algebraic structures discussed in the present work have a more natural setting in the language of superalgebras. We have not investigated this question. Another interesting question is whether the dynamical symmetries considered in this paper are related to fermionic Killing-Yano supercharges [20]. This problem needs further investigations. Finally, we see no obstacle against extending our method to the scattering problem of the supersymmetric hydrogen atom, for which the dynamical symmetry group $S O(1, d)$ is non-compact.

\section{Acknowledgments}

We thank Falk Bruckmann for his collaboration at an early stage of this project and Thomas Strobl and Jan-Willem van Holten for useful discussions. P.A.G. Pisani thanks people at the TPI for their kind hospitality. A. Kirchberg and P.A.G. Pisani are supported by the Studienstiftung des Deutschen Volkes and CONICET, respectively. This project has in part been supported by Foundación Antorchas and DAAD (Grant 13887/1-87). Most group-theoretical calculations have been performed with the powerful package LiE [19].

\section{Appendix A. Representations of rotation groups}

In this appendix we collect the group theoretical facts needed in the main body of the paper (cf. [14,16-18] for a more detailed discussion of these issues). We shall construct the relevant irreducible representations of the total angular momentum operators

$$
J_{a b}=\frac{1}{\mathrm{i}}\left(x_{a} \frac{\partial}{\partial x_{b}}-x_{b} \frac{\partial}{\partial x_{a}}\right)+\frac{1}{\mathrm{i}}\left(\psi_{a}^{\dagger} \psi_{b}-\psi_{b}^{\dagger} \psi_{a}\right), \quad a, b=1, \ldots, d,
$$

satisfying the $s o(d)$ commutation relations

$$
\left[J_{a b}, J_{c d}\right]=\mathrm{i}\left(\delta_{a c} J_{b d}+\delta_{b d} J_{a c}-\delta_{a d} J_{b c}-\delta_{b c} J_{a d}\right)
$$

on wave functions in

$$
\mathscr{H}_{p}=L_{2}\left(\mathbb{R}^{d}\right) \times \mathbb{C}^{\left(\begin{array}{l}
d \\
p
\end{array}\right)} \quad \text { with } p=0, \ldots, d .
$$

The fermionic operators $\psi_{a}$ have been introduced earlier in Section 3. It is convenient to use the Cartan-Weyl basis consisting of generators $H_{i}$ in the Cartan subalgebra and one raising and one lowering operator $E_{\alpha}$ and $E_{-\alpha}$ for every positive root $\alpha$,

$$
\left[H_{i}, E_{\alpha}\right]=\alpha_{i} E_{\alpha} \text { and }\left[E_{\alpha}, E_{-\alpha}\right]=\alpha \cdot H \text { with } E_{-\alpha}=E_{\alpha}^{\dagger} \text {. }
$$

Because of their different properties we do this separately for the groups $D_{n} \sim S O(2 n)$ and $B_{n} \sim S O(2 n+1)$. 


\section{A.1. Total angular momentum for the $S O(d=2 n)$ groups}

To proceed it is very convenient to introduce complex coordinates in $\mathbb{R}^{2 n}$,

$$
\begin{aligned}
& z_{i}=\frac{1}{\sqrt{2}}\left(x_{2 i-1}+\mathrm{i} x_{2 i}\right), \quad \bar{z}_{i}=\frac{1}{\sqrt{2}}\left(x_{2 i-1}-\mathrm{i} x_{2 i}\right), \\
& \partial_{i}=\frac{1}{\sqrt{2}}\left(\partial_{x_{2 i-1}}-\mathrm{i} \partial_{x_{2 i}}\right), \quad \bar{\partial}_{i}=\frac{1}{\sqrt{2}}\left(\partial_{x_{2 i-1}}+\mathrm{i} \partial_{x_{2 i}}\right), \quad i=1, \ldots, n,
\end{aligned}
$$

and similarly two sets of complex creation and annihilation operators

$$
\begin{aligned}
& \phi_{i}^{\dagger}=\frac{1}{\sqrt{2}}\left(\psi_{2 i-1}^{\dagger}+\mathrm{i} \psi_{2 i}^{\dagger}\right), \quad \bar{\phi}_{i}^{\dagger}=\frac{1}{\sqrt{2}}\left(\psi_{2 i-1}^{\dagger}-\mathrm{i} \psi_{2 i}^{\dagger}\right), \\
& \phi_{i}=\frac{1}{\sqrt{2}}\left(\psi_{2 i-1}-\mathrm{i} \psi_{2 i}\right), \quad \bar{\phi}_{i}=\frac{1}{\sqrt{2}}\left(\psi_{2 i-1}+\mathrm{i} \psi_{2 i}\right), \quad i=1, \ldots, n .
\end{aligned}
$$

The only non-vanishing anticommutators are

$$
\left\{\phi_{i}, \phi_{j}^{\dagger}\right\}=\left\{\bar{\phi}_{i}, \bar{\phi}_{j}^{\dagger}\right\}=\delta_{i j}
$$

The generators in the Cartan subalgebra take the simple form

$$
H_{i}=J_{2 i-1,2 i}=z_{i} \partial_{i}-\bar{z}_{i} \bar{\partial}_{i}+\phi_{i}^{\dagger} \phi_{i}-\bar{\phi}_{i}^{\dagger} \bar{\phi}_{i}, \quad i=1, \ldots, n,
$$

and there are two types of raising operators:

$$
\begin{aligned}
& E_{\alpha}=\frac{1}{2}\left(J_{2 i-1,2 j-1}+J_{2 i, 2 j}-\mathrm{i} J_{2 i-1,2 j}+\mathrm{i} J_{2 i, 2 j-1}\right) \quad \text { with root } \alpha=e_{i}-e_{j}, \\
& E_{\alpha}=\frac{1}{2}\left(J_{2 i-1,2 j-1}-J_{2 i, 2 j}+\mathrm{i} J_{2 i-1,2 j}+\mathrm{i} J_{2 i, 2 j-1}\right) \quad \text { with root } \alpha=e_{i}+e_{j},
\end{aligned}
$$

where $i<j$ is assumed. In terms of the complex coordinates/operators they read

$$
\begin{aligned}
& E_{\alpha}=\frac{1}{\mathrm{i}}\left(z_{i} \partial_{j}-\bar{z}_{j} \bar{\partial}_{i}+\phi_{i}^{\dagger} \phi_{j}-\bar{\phi}_{j}^{\dagger} \bar{\phi}_{i}\right) \quad \text { with } \operatorname{root} \alpha=e_{i}-e_{j}, \\
& E_{\alpha}=\frac{1}{\mathrm{i}}\left(z_{i} \bar{\partial}_{j}-z_{j} \bar{\partial}_{i}+\phi_{i}^{\dagger} \bar{\phi}_{j}-\phi_{j}^{\dagger} \bar{\phi}_{i}\right) \quad \text { with } \operatorname{root} \alpha=e_{i}+e_{j} .
\end{aligned}
$$

The corresponding lowering operators are just the adjoints of the raising operators. The operators $\left(H_{i}, E_{\alpha}, E_{-\alpha}\right)$ satisfy the commutation relations (A.1) with corresponding positive roots in (A.5). The $n$ simple roots are

$$
\alpha_{i}=e_{i}-e_{i+1}, \quad 1 \leqslant i<n \text { and } \alpha_{n}=e_{n-1}+e_{n},
$$

and the corresponding raising operators have the form

$$
\begin{aligned}
& E_{i}=\frac{1}{\mathrm{i}}\left(z_{i} \partial_{i+1}-\bar{z}_{i+1} \bar{\partial}_{i}+\phi_{i}^{\dagger} \phi_{i+1}-\bar{\phi}_{i+1}^{\dagger} \bar{\phi}_{i}\right), \quad \alpha=e_{i}-e_{i+1}, 1 \leqslant i<n \\
& E_{n}=\frac{1}{\mathrm{i}}\left(z_{n-1} \bar{\partial}_{n}-z_{n} \bar{\partial}_{n-1}+\phi_{n-1}^{\dagger} \bar{\phi}_{n}-\phi_{n}^{\dagger} \bar{\phi}_{n-1}\right), \quad \alpha=e_{n-1}+e_{n} .
\end{aligned}
$$


With the help of the Weyl vector

$$
\delta=\frac{1}{2} \sum_{\alpha>0} \alpha=(n-1) e_{1}+(n-2) e_{2}+\cdots+e_{n-1},
$$

where the sum extends over all positive roots in (A.5), we may calculate the dimension of an arbitrary faithful representation of $S O(2 n)$. Such a representation is determined by its Young tableau containing at most $n$ rows. The length $\ell_{i}$ of row $i$ is bigger or equal to that of row $i+1$. Hence, a Young tableau is given by $n$ nonnegative ordered integers

$$
\ell_{1} \geqslant \ell_{2} \geqslant \cdots \geqslant \ell_{n-1} \geqslant \ell_{n},
$$

and has the form

$$
p\left\{\begin{array}{c|c|c|c|c|c}
\hline 1 & 2 & \cdot & \cdot & \cdot & \ell_{1} \\
\hline 1 & 2 & \cdot & \cdot & \ell_{2} \\
\hline: & : & : & : &
\end{array} \quad, \quad p \leq n\right.
$$

Rows with length 0 are not shown when one draws a Young tableau. The corresponding representation $\mathscr{D}^{\ell_{1}, \ldots, \ell_{n}}$ has the dimension

$$
\operatorname{dim}\left(\mathscr{D}^{\ell_{1} \ldots \ell_{n}}\right)=\prod_{1 \leqslant r<s \leqslant n} \frac{\ell_{r}+\ell_{s}+2 n-r-s}{2 n-r-s} \frac{\ell_{r}-\ell_{s}+s-r}{s-r} .
$$

For the second-order Casimir invariant of these representations one obtains the formula

$$
\mathscr{C}_{(2)}\left(\mathscr{D}^{\ell_{1} \ldots \ell_{n}}\right)=\sum_{r} \ell_{r}\left(\ell_{r}+2 n-2 r\right) .
$$

In particular, for the completely symmetric representations

$$
\mathcal{D}^{\ell 0 \ldots 0} \equiv \mathcal{D}_{1}^{\ell} \sim \begin{array}{|l|l|l|l|l|}
\hline 1 & 2 & \cdot & \cdot & \cdot \\
\hline
\end{array}
$$

these formulae simplify to

$$
\mathscr{C}_{(2)}\left(\mathscr{D}_{1}^{\ell}\right)=\ell(\ell+d-2) \quad \text { and } \quad \operatorname{dim}\left(\mathscr{D}_{1}^{\ell}\right)=\left(\begin{array}{c}
\ell+d-1 \\
\ell
\end{array}\right)-\left(\begin{array}{c}
\ell+d-3 \\
\ell-2
\end{array}\right) .
$$

For the completely antisymmetric representations

$$
\mathcal{D}^{1,1, \ldots 1} \equiv \mathcal{D}_{p}^{1} \sim \frac{1}{\frac{1}{:}}
$$

they simplify to

$$
\mathscr{C}_{(2)}\left(\mathscr{D}_{p}^{1}\right)=p(d-p) \quad \text { and } \quad \operatorname{dim}\left(\mathscr{D}_{p}^{1}\right)=\left(\begin{array}{l}
d \\
p
\end{array}\right) .
$$


Simultaneous eigenstates of all $n$ generators $H_{i}$ in the Cartan subalgebra have the form

$$
\prod_{i=1}^{n} z_{i}^{m_{i}} \bar{z}_{i}^{\bar{m}_{i}}\left|\vec{p} \vec{p}^{\prime}\right\rangle, \quad\left|\vec{p} \vec{p}^{\prime}\right\rangle=\phi_{1}^{\dagger p_{1}} \ldots \phi_{n}^{\dagger p_{n}} \bar{\phi}_{1}^{\dagger p_{1}^{\prime}} \ldots \bar{\phi}_{n}^{\dagger p_{n}^{\prime}}|0\rangle,
$$

where $m_{i}, \bar{m}_{i} \in \mathbb{N}_{0}$ and $p_{i}, p_{i}^{\prime} \in\{0,1\}$. The vacuum $|0\rangle$ is annihilated by all particle lowering operators $\psi_{a}$ or equivalently by all $\phi_{i}$ and $\bar{\phi}_{i}$. The $H_{i}$-eigenvalues of these states are $m_{i}-\bar{m}_{i}+p_{i}-p_{i}^{\prime}$.

Next we must construct the highest weight states which are annihilated by all raising operators. Every such state determines an irreducible representation. The eigenvalues of $H_{i}$ on a highest weight state is equal to the length $\ell_{i}$ of the Young tableau corresponding to the irreducible representation determined by this weight. The $d+2$ space-independent highest weight states are

$$
|p\rangle=\left|\vec{p} \vec{p}^{\prime}\right\rangle \quad \text { with } p_{1} \geqslant \cdots \geqslant p_{n} \geqslant p_{n}^{\prime} \geqslant \cdots \geqslant p_{1}^{\prime} \text { and } \sum\left(p_{i}+p_{i}^{\prime}\right)=p .
$$

There is an additional highest weight state in the $p=n$ particle sector, that arises since in this sector we have selfdual and anti-selfdual configurations. It is given by

$$
p_{1}=\cdots=p_{n-1}=p_{n}^{\prime}=1, \quad p_{n}=p_{1}^{\prime}=\cdots=p_{n-1}^{\prime}=0 .
$$

Clearly, the particle number $p$ uniquely specifies these states since the $p_{i}, p_{i}^{\prime}$ s are ordered. These states define the completely antisymmetric representations

$$
\mathscr{D}_{p}^{1} \text { for } p \leqslant n \text { and } \mathscr{D}_{p}^{1} \sim \mathscr{D}_{2 n-p}^{1} \text { for } p \geqslant n \text {. }
$$

We used that a Young tableau, the first column of which has length $n \leqslant p \leqslant 2 n$, gives rise to the same multiplet as the tableau with first column of length $2 n-p \leqslant n$. In the following one should replace $\mathscr{D}_{p}^{\ell}$ by $\mathscr{D}_{2 n-p}^{\ell}$ if $p$ exceeds $n$. Also note that $\mathscr{D}_{0}^{1} \sim \mathscr{D}_{d}^{1}$ is the one-dimensional trivial representation.

The highest weight states in the zero-particle sector are

$$
z_{1}^{\ell}|0\rangle
$$

and they give rise to the completely symmetric representations $\mathscr{D}_{1}^{\ell}$ spanned by the harmonic polynomials of order $\ell$. The relevant irreducible representation of $S O(2 n)$ in the $p$-particle sector is gotten by tensoring the antisymmetric representation $\mathscr{D}_{p}^{1}$ with a symmetric representation $\mathscr{D}_{1}^{\ell}$. We use

$$
\mathscr{D}_{p}^{1} \otimes \mathscr{D}_{1}^{\ell}=\mathscr{D}_{p-1}^{\ell} \oplus \mathscr{D}_{p}^{\ell-1} \oplus \mathscr{D}_{p}^{\ell+1} \oplus \mathscr{D}_{p+1}^{\ell}
$$

or in the language of Young tableaux

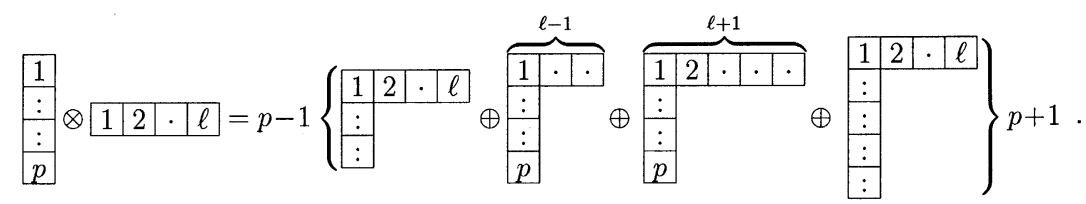

Note that for $p=1$ and/or $\ell=1$ there appear only three representations in this decomposition. For $p=1$ the first representation and for $\ell=1$ the second 
representation on the right-hand side in (A.11) are absent. Also note that for $p=n$ the first and last representations are equivalent. The second to last representation $\mathscr{D}_{p}^{\ell+1}$ on the right-hand side has highest weight state

$$
\mathscr{Y}_{s}(\ell+1, p)=z_{1}^{\ell}|p\rangle,
$$

as it is the product of the highest weight states of $\mathscr{D}_{p}^{1}$ and $\mathscr{D}_{1}^{\ell}$. To find the highest weight state of the other representations we observe that the operators

$$
r S=x_{a} \psi_{a}=z_{i} \phi_{i}+\bar{z}_{i} \bar{\phi}_{i} \text { and } r S^{\dagger}=x_{a} \psi_{a}^{\dagger}=\bar{z}_{i} \phi_{i}^{\dagger}+z_{i} \bar{\phi}_{i}^{\dagger},
$$

which have been introduced in (35), commute with the total angular momentum and hence map highest weight states into highest weight states. Since $S$ decreases and $S^{\dagger}$ increases the particle number by one, we find the state

$$
\mathscr{Y}_{a}(\ell, p+1)=r S \mathscr{Y}_{s}(\ell, p+1)=\sum_{i=1}^{p+1}(-)^{i+1} z_{i} \phi_{1}^{\dagger} \ldots \check{\phi}_{i}^{\dagger} \ldots \phi_{p+1}^{\dagger} z_{1}^{\ell-1}|0\rangle,
$$

which is highest weight state of the last representation $\mathscr{D}_{p+1}^{\ell}$ in the decomposition (A.11). The missing two highest weight states correspond to those representations in the tensor product of a symmetric and an antisymmetric representation which one obtains by taking the trace over two suitable indices. This operation is equivalent to acting with $S^{\dagger}$. Thus

$$
\mathscr{T}_{s}(\ell, p-1)=S^{\dagger} \mathscr{Y}_{s}(\ell, p-1)
$$

is the highest weight state of $\mathscr{D}_{p-1}^{\ell}$ in the decomposition (A.11). For the remaining highest weight state we make the ansatz

$$
\mathscr{T}_{a}(\ell-1, p)=\left(S S^{\dagger}+\alpha S^{\dagger} S\right) \mathscr{Y}_{s}(\ell-1, p) .
$$

As $\left\{S, S^{\dagger}\right\}=1$ this state may have a component in the direction of $\mathscr{Y}_{s}(\ell-1, p)$. However, for the choice $\alpha=-1$ the highest weight state

$$
\mathscr{T}_{a}(\ell-1, p)=\left[S, S^{\dagger}\right] \mathscr{Y}_{s}(\ell-1, p)
$$

is orthogonal to $\mathscr{Y}_{s}(\ell-1, p)$.

\section{A.2. Total angular momentum for the $S O(d=2 n+1)$ groups}

The rotation group $S O(2 n+1)$ has the same rank as its subgroup $S O(2 n)$ and hence we may still use the Cartan generators (A.4), i.e.,

$$
H_{i}=J_{2 i-1,2 i}=z_{i} \partial_{i}-\bar{z}_{i} \bar{\partial}_{i}+\phi_{i}^{\dagger} \phi_{i}-\bar{\phi}_{i}^{\dagger} \bar{\phi}_{i}, \quad i=1, \ldots, n .
$$

We use the complex coordinates (A.2) and the complex creation- and annihilation operators (A.3), supplemented by the last coordinate $x_{d}$ and the last creation and annihilation operator $\psi_{d}^{\dagger}$ and $\psi_{d}$. Clearly, the raising operators (A.5) are still raising operators of $s o(2 n+1)$ with the same positive roots. But since

$$
\operatorname{dim}(S O(2 n+1))=\operatorname{dim}(S O(2 n))+2 n \text { and } \operatorname{rank}(S O(2 n+1))=\operatorname{rank}(S O(2 n))
$$


there are $n$ positive roots missing. These are

$$
E_{\alpha}=\frac{1}{\sqrt{2}}\left(J_{2 i-1, d}+\mathrm{i} J_{2 i, d}\right)=\frac{1}{\mathrm{i}}\left(z_{i} \partial_{x_{d}}-x_{d} \bar{\partial}_{i}+\phi_{i}^{\dagger} \psi_{d}-\psi_{d}^{\dagger} \bar{\phi}_{i}\right), \quad \alpha=e_{i},
$$

where $1 \leqslant i \leqslant n$. The first $n-1$ simple roots are the same as in (A.6), but the last one is replaced by $e_{n}$. Hence the raising operators corresponding to the simple roots read

$$
\begin{aligned}
& E_{i}=\frac{1}{\mathrm{i}}\left(z_{i} \partial_{i+1}-\bar{z}_{i+1} \bar{\partial}_{i}+\phi_{i}^{\dagger} \phi_{i+1}-\bar{\phi}_{i+1}^{\dagger} \bar{\phi}_{i}\right), \quad \alpha=e_{i}-e_{i+1}, \quad 1 \leqslant i<n, \\
& E_{n}=\frac{1}{\mathrm{i}}\left(z_{n} \partial_{x_{d}}-x_{d} \bar{\partial}_{n}+\phi_{n}^{\dagger} \psi_{d}-\psi_{d}^{\dagger} \bar{\phi}_{n}\right), \quad \alpha=e_{n} .
\end{aligned}
$$

The Young tableaux are identical to those of $S O(2 n)$ and hence are characterized by $n$ ordered non-negative integers $\ell_{1}, \ldots, \ell_{n}$. The dimensions of the corresponding representations read

$$
\begin{aligned}
\operatorname{dim}\left(\mathscr{D}^{\ell_{1} \ldots \ell_{n}}\right)= & \prod_{t=1}^{n} \frac{2 \ell_{t}+d-2 t}{d-2 t} \prod_{1 \leqslant r<s \leqslant n} \\
& \times \frac{\ell_{r}+\ell_{s}+d-r-s}{d-r-s} \frac{\ell_{r}-\ell_{s}+s-r}{s-r},
\end{aligned}
$$

and the formula for the second-order Casimir is the same as for the $s o(2 n)$ algebra

$$
C_{(2)}\left(\mathscr{D}^{\ell_{1} \ldots \ell_{n}}\right)=\sum_{r} \ell_{r}\left(\ell_{r}+d-2 r\right) \text {. }
$$

Also the rules for tensor products are identical to those of $S O(2 n)$.

Since the simple roots are different, the highest weight states have a slightly different form. The simultaneous eigenstates of the $n$ generators in the Cartan subalgebra read

$$
f\left(x_{d}\right) \prod_{i} z_{i}^{m_{i}} \bar{z}_{i}^{\bar{m}_{i}}\left|\vec{p} q \vec{p}^{\prime}\right\rangle, \quad\left|\vec{p} q \vec{p}^{\prime}\right\rangle=\phi_{1}^{\dagger p_{1}} \ldots \phi_{n}^{\dagger p_{n}} \psi_{d}^{\dagger q} \bar{\phi}_{1}^{\dagger p_{1}^{\prime}} \ldots \bar{\phi}_{n}^{\dagger p_{n}^{\prime}}|0\rangle,
$$

where $m_{i}, \bar{m}_{i} \in \mathbb{Z}$ and $p_{i}, q, \bar{p}_{i} \in\{0,1\}$. The $d+1$ constant highest weight states are

$$
|p\rangle=\left|\vec{p} q \vec{p}^{\prime}\right\rangle \quad \text { with } p_{1} \geqslant \cdots \geqslant p_{n} \geqslant q \geqslant p_{n}^{\prime} \geqslant \cdots \geqslant p_{1}^{\prime},
$$

where $p=\sum\left(p_{i}+p_{i}^{\prime}\right)+q$ denotes the particle number. The highest weight of $\mathscr{D}_{p}^{\ell+1}$ in the decomposition

$$
\mathscr{D}_{p}^{1} \otimes \mathscr{D}_{1}^{\ell}=\mathscr{D}_{p-1}^{\ell} \oplus \mathscr{D}_{p}^{\ell-1} \oplus \mathscr{D}_{p}^{\ell+1} \oplus \mathscr{D}_{p+1}^{\ell}
$$

is again determined by the highest weight state

$$
\mathscr{Y}_{s}(\ell+1, p)=z_{1}^{\ell}|p\rangle \text {. }
$$

As in even dimensions one may use the scalar operators

$$
r S=x_{a} \psi_{a}=z_{i} \phi_{i}+\bar{z}_{i} \bar{\phi}_{i}+x_{d} \psi_{d} \quad \text { and } \quad r S^{\dagger}=x_{a} \psi_{a}^{\dagger}=\bar{z}_{i} \phi_{i}^{\dagger}+z_{i} \bar{\phi}_{i}^{\dagger}+x_{d} \psi_{d}^{\dagger}
$$

to obtain the highest weight states,

$$
\mathscr{Y}_{a}(\ell, p+1)=r S \mathscr{Y}_{s}(\ell, p+1) \rightarrow \mathscr{D}_{p+1}^{\ell},
$$




$$
\begin{aligned}
& \mathscr{T}_{s}(\ell, p-1)=S^{\dagger} \mathscr{Y}_{s}(\ell, p-1) \rightarrow \mathscr{D}_{p-1}^{\ell}, \\
& \mathscr{T}_{a}(\ell-1, p)=\left[S, S^{\dagger}\right] \mathscr{Y}_{s}(\ell-1, p) \rightarrow \mathscr{D}_{p}^{\ell-1},
\end{aligned}
$$

of the remaining irreducible representations in (A.18).

\section{Appendix B. Rotation groups vs. dynamical symmetry groups}

In the main body of the paper we have seen that the total angular momentum $J_{a b}$ in (33) together with $K_{a}$ in (40) combine to generators of the dynamical symmetry group $S O(d+1)$,

$$
J_{A B}=\left(\begin{array}{c|c}
J_{a b} & K_{a} \\
\hline-K_{b} & 0
\end{array}\right) .
$$

The rotational group with generators $J_{a b}$ discussed in the previous part of the appendix must be embedded into the dynamical group

$$
\begin{aligned}
& d=2 n: S O(2 n) \subset S O(2 n+1), \\
& d=2 n+1: S O(2 n+1) \subset S O(2 n+2) .
\end{aligned}
$$

Even dimensions: The dynamical symmetry group has the same rank as the rotation group $S O(2 n)$ and we can repeat our construction in Appendix A.2, where we extended $S O(2 n)$ to $S O(2 n+1)$. Of course we should take into account that the components in the last column and last row of $\left(J_{A B}\right)$ are the components of $K_{a}$. The Cartan generators are those in (A.14) and the first $n-1$ raising operators are given in (A.15). But the last raising operator (A.16) is of course replaced by

$$
E_{n}=\frac{1}{\sqrt{2}}\left(K_{d-1}+\mathrm{i} K_{d}\right)
$$

which is proportional to $\frac{1}{\sqrt{2}}\left(C_{d-1}+\mathrm{i} C_{d}\right)$. The latter has been given in (48).

Odd dimensions: The rank of the dynamical symmetry group $S O(2 n+2)$ exceeds the rank of the rotation group $S O(2 n+1)$ by 1 . The Cartan generators are given by the $n$ operators $H_{i}$ in (A.4), supplemented by $H_{n+1}=K_{d} \sim C_{d}$, where the explicit realization of $C_{d}$ is given in (50). The raising operators are the $n-1$ operators $E_{i}$ in (A.15) plus the two operators

$$
\begin{aligned}
& E_{\alpha}=\frac{1}{2}\left(J_{d-2, d}+K_{d-1}-\mathrm{i} K_{d-2}+\mathrm{i} J_{d-1, d}\right), \quad \alpha=e_{n}-e_{n+1}, \\
& E_{\alpha}^{\prime}=\frac{1}{2}\left(J_{d-2, d}-K_{d-1}+\mathrm{i} K_{d-2}+\mathrm{i} J_{d-1, d}\right), \quad \alpha=e_{n}+e_{n+1} .
\end{aligned}
$$

Highest weight states are annihilated by these two raising operators and it is convenient to use two (independent) combinations of these operators, namely the operators 


$$
\frac{1}{\sqrt{2}}\left(E_{\alpha}+E_{\alpha}^{\prime}\right)=E_{n} \quad \text { and } \quad \frac{\mathrm{i}}{\sqrt{2}}\left(E_{\alpha}-E_{\alpha}^{\prime}\right) \sim \frac{1}{\sqrt{2}}\left(C_{d-2}+\mathrm{i} C_{d-1}\right)=E_{n+1} .
$$

Their explicit forms can be found in (48) and (51).

\section{References}

[1] J. Hermann, G. Letterati D'Italia 2 (1710) 447;

J. Bernoulli, Histoires de L'Academie Royale des Sciences avec les Mémoires de Mathematique et Physique (1712) 523;

P.S. de Laplace, Traité de mécanique celeste Paris An VII, pp. 1798-1799;

C. Runge, Vektoranalysis, vol. 1, Hirzel, Leipzig, 1919;

W. Lenz, Z. Phys. 24 (1924) 197.

[2] H. Goldstein, Am. J. Phys. 43 (1975) 737; Am. J. Phys. 44 (1976) 1123.

[3] W. Pauli, Z. Phys. 36 (1926) 336;

L. Hulthén, Z. Phys. 86 (1933) 21;

V. Bargmann, Z. Phys. 99 (1936) 576.

[4] V. Fock, Z. Phys. 98 (1935) 145.

[5] D. Zwanziger, J. Math. Phys. 8 (1967) 1858.

[6] S.P. Alliluev, Soviet Phys. JETP 6 (1958) 156.

[7] M. Bander, C. Itzykson, Rev. Modern Phys. 38 (1966) 330.

[8] J.F. Cornwell, Group Theory in Physics, vol. 2, Academic Press, London, 1984.

[9] E. Witten, J. Differential Geom. 17 (1982) 661.

[10] E. Witten, Nucl. Phys. B 188 (1981) 513.

[11] H. Nicolai, J. Phys. A 9 (1976) 1497.

[12] F. Cooper, A. Khare, R. Musto, A. Wipf, Ann. Phys. 187 (1988) 1.

[13] A.A. Andrianov, N.V. Borisov, M.V. Ioffe, Phys. Lett. A 105 (1984) 19.

[14] W.G. McKay, J. Patera, Tables of Dimensions, Indices, and Branching Rules for Representations of Simple Lie Algebras, Lecture Notes in Pure and Appl. Math., vol. 69, Dekker, New York, 1981.

[15] R. Heumann, J. Phys. A 35 (2002) 7437.

[16] M. Hamermesh, Group Theory and its Application to Physical Problems, Dover, New York, 1962.

[17] J.E. Humphreys, Introduction to Lie Algebras and Representation Theory, Springer, New York, 1972.

[18] V.S. Varadarajan, Lie Groups, Lie Algebras, and their Representations, Graduate Texts in Mathematics, vol. 102, Springer, New York, 1984.

[19] M. van Leeuwen, A. Cohen, B. Lisser, LiE 2.1 Manual, Computer Algebra Group of CWI, Amsterdam.

[20] G.W. Gibbons, R.H. Rietdijk, J.W. van Holten, Nucl. Phys. B 404 (1993) 42.

[21] E.C.G. Sudarshan, N. Mukunda, L. O'Raifeartaigh, Phys. Lett. 19 (1965) 322. 